\title{
Characterization of open cluster remnants ${ }^{\star}$
}

\author{
D. B. Pavani ${ }^{1,2}$ and E. Bica ${ }^{2}$ \\ 1 Instituto de Astronomia, Geofísica e Ciências Atmosféricas (IAG) Universidade de São Paulo - Rua do Matão 1226, 05508-900, \\ São Paulo, SP, Brazil \\ e-mail: daniela@astro.iag.usp.br \\ 2 Universidade Federal do Rio Grande do Sul, IF, CP 15051, 912501-970, Porto Alegre, RS, Brazil \\ e-mail: bica@if.ufrgs.br
}

Received 11 August 2006 / Accepted 23 January 2007

ABSTRACT

\begin{abstract}
Context. Despite progress in the theoretical knowledge of open cluster remnants and the growing search for observational identifications in recent years, open questions still remain. The methods used to analyze open cluster remnants and criteria to define them as physical systems are not homogeneous. In this work we present a systematic method for studying these objects that provides a view of their properties and allows their characterization.

Aims. Eighteen remnant candidates are analyzed by means of photometric and proper motion data. These data provide information on objects and their fields. We establish criteria for characterizing open cluster remnants, taking observational uncertainties into account. Methods. 2MASS J and H photometry is employed (i) to study structural properties of the objects by means of radial stellar density profiles, (ii) to test for any similarity between objects and fields with a statistical comparison method applied to the distributions of stars in the CMDs, and (iii) to obtain ages, reddening values, and distances from the CMD, taking an index of isochrone fit into account. The UCAC2 proper motions allowed an objective comparison between objects and large solid angle offset fields.

Results. The objective analysis based on the present methods indicates 13 open-cluster remnants in the sample. Evidence of the presence of binary stars is found, as expected for dynamically evolved systems. Finally, we infer possible evolutionary stages among remnants from the structure, proper motion, and CMD distributions. The low stellar statistics for individual objects is overcome by means of the construction of composite proper motion and CMD diagrams. The distributions of remnants in the composite diagrams resemble the single-star and unresolved binary star distributions of open clusters.
\end{abstract}

Key words. Galaxy: open clusters and associations: general - methods: observational

\section{Introduction}

The study of the space distribution, formation, age, and structure of open clusters provides information on their evolution. Open clusters are destroyed over time by the action of both internal forces (mass loss through the dynamical evolution and stellar evolution) and external ones, such as interactions with the Galactic tidal field, collisions with molecular clouds, and/or disc shocking (Friel 1999; Wielen 1971). Portegies Zwart et al. (2002) confirm central Galaxy tidal-field effects by showing that star clusters located inside $150 \mathrm{pc}$ of the Galactic center can be dissolved in 50 Myr. In the solar neighborhood, most open clusters evaporate completely in less than 1 Gyr (Bergond et al. 2001; Bonatto \& Bica 2005). However, older objects exist (Lyngå 1995; Dutra \& Bica 2000). By means of the open cluster catalogue by Dias et al. (2002), updated to January $2006^{1}$, we concluded that objects older than 1 Gyr amount to $\approx 10 \%$ of the 864 clusters with available ages.

Open cluster remnants (hereafter, OCR) correspond to final evolutionary stages. Numerical simulations (Terlevich 1987; de La Fuente Marcos 1997, 1998) have shown that the final stellar content of OCRs depends on their initial mass function, fraction of primordial binaries, Galactocentric distance, and total mass. The simulations also show that a strong dependence exists

\footnotetext{
* Figures 3, 7, 9 and 10 are only available in electronic form at http://www . aanda.org

${ }^{1}$ http://www.astro.iag.usp.br/ wilton
}

between the dissolution timescale of an open cluster and the stellar mass evaporation. As a consequence, OCRs are expected to be rich in binary stars. Owing to dynamical processes that dominate the dissolution, it is reasonable to assume that the effects of mass segregation will cause depletion of the low main sequence (MS) in OCRs. Considering the above results with those of Hsu et al. (1996), Friel (1999) made further developments towards an evolutionary scenario. Small and poorly populated open clusters dissolve primarily from internal dynamical effects on the order of a few $10^{8} \mathrm{Myr}$, which explains most of the open cluster population. Apparently there is no relationship between dissolution rate and location in the Galaxy for low-mass open clusters. For richer and more massive open clusters, the Galactic field effects become significant. Those with intermediate masses ( $\sim 500$ to $1000 M_{\odot}$ ) can survive several Gyr, if they are located in the external regions of the disc (e.g., NGC 752 and NGC 3680). Open clusters that survive longer (e.g. NGC 6791) must be more massive or else located exclusively in external regions of the disc (NGC 188), or both.

From an observational point of view, an OCR can be defined as a poorly populated concentration of stars resulting from the dynamical evolution of a more massive system (Pavani et al. 2003). Despite theoretical progress, and the growing number of observed candidates, open questions still remain, such as: (i) is there a preferential location in the Galaxy for OCRs? (ii) Do different evolutionary stages exist among remnants? (iii) Is it possible to define criteria for the characterization of OCRs? 
Studies in the literature reflect the debate on the observational evidence of OCRs. For instance, NGC 6994 (M73) was interpreted as a possible remnant of an old open cluster by means of high-resolution spectra studies, radial velocities, atmospheric parameters, and proper motions (Bassino et al. 2000). On the other hand, Carraro (2000) and Odenkirchen \& Soubiran (2002) interpret NGC 6994 as a random fluctuation of the stellar density using CCD photometry in $B, V$, and $I$ bands and high-resolution spectra of the six brightest stars, respectively.

Bica et al. (2001) present 34 possible open cluster remnants (POCR) that are located at relatively high galactic latitudes $\left(|b|>15^{\circ}\right)$ and are underpopulated with respect to the usual open clusters. They show a significant density contrast of brighter stars as compared to the Galactic field. Among the objects in that list, some have already been studied in detail it was concluded that NGC 1252 is a remnant at $d_{\odot}=0.64 \mathrm{kpc}$ from the Sun, with an age of $3 \pm 1$ Gyr. NGC 1901 is a physical system dynamically comparable to the Hyades, at $d_{\odot}=0.45 \mathrm{kpc}$, and with an age of $0.6 \pm 0.1 \mathrm{Gyr}$. Both were studied with $B, V$ photometry, from the Tycho-2's proper motions and comparisons of object CMDs with model predictions for the Galactic field (Pavani et al. 2001). Carraro (2002) concludes that NGC 7772 and NGC 7036 are remnants. NGC 7772 is located at $d_{\odot}=1 \mathrm{kpc}$, with 14 members and an age of 1.5 Gyr. NGC 7036 has 17 members and is located at $d_{\odot}=1 \mathrm{kpc}$ with an age of 3-4 Gyr. He used UBVI photometry for color-color diagrams, CMD, and a radial stellar density profile. NGC 1663 was found to be a remnant of 2 Gyr located at $d_{\odot}=0.7 \mathrm{kpc}$ (Baume et al. 2003). They employed high-resolution spectra of the brightest stars and 2MASS photometry (Skrutskie et al. 1997) ${ }^{2}$, together with Tycho-2's astrometric data. Pavani et al. (2003) find that Ruprecht 3 is a remnant at $d_{\odot}=0.72 \mathrm{kpc}$ with an age of $1.5 \pm 0.5 \mathrm{Gyr}$, using $J$ and $H$ 2MASS photometry, integrated and individual star spectroscopy, and statistical methods for subtracting field stars from the CMD. Bonatto et al. (2004) presented NGC 2180 as a probable missing link between evolved open clusters and remnants using $J$ and $H$ 2MASS photometry. They discuss luminosity and mass functions, structure, and CMDs. They obtained an age of $700 \pm 70 \mathrm{Myr}, d_{\odot}=0.91 \pm$ $0.08 \mathrm{kpc}$, and observed mass $m_{\mathrm{obs}} \sim 47 M_{\odot}$. Villanova et al. (2004) obtained CCD UBVI photometry and medium/high resolution spectroscopy for NGC 5385, NGC 2664 and Collinder 21 and their fields. The analysis using star counts, photometry, radial velocity distribution, and Tycho-2's proper motions indicated they are non physical objects. Carraro et al. (2005) discuss the nature of 11 possible OCRs by using the Southern Proper Motion (SPM) Program 3 combined with 2MASS photometry. They conclude that ESO 282 SC26 is a probable physical group.

The methods employed in the series of studies above are not homogeneous. The present study intends to characterize OCRs by using a systematic set of observational methods applied to a large sample of candidates. We include new POCRs and objects in common with previous studies. This is essential for establishing criteria for the observational identification and determination of OCR properties, taking observational uncertainties into account.

In Sect. 2 we present the criteria for object selection and their structural properties. In Sect. 3 we describe a statistical comparison test between distributions of stars in the CMD. In Sect. 4 we discuss the $J \times(J-H)$ CMDs and derive ages, reddening values, and distances, together with a classification of the objects based

\footnotetext{
2 Two Micron All Sky Survey, All Sky data release, available at http://www . ipac . caltech. edu/2mass/releases/allsky
}

on an index of isochrone fitting. In Sect. 5 we present the proper motion analysis. In Sect. 6 we characterize the OCRs. Finally, concluding remarks are given in Sect. 7.

\section{Methods}

\subsection{Object selection}

The selection of POCRs was based on poorly populated stellar concentrations included in the catalogues by Alter et al. (1970) and Lyngå (1995). The objects have a significant stellar density contrast as compared to the background. Among the initially selected objects, we kept those presenting evidence of evolutionary sequences in the CMD. We favored higher Galactic latitudes to avoid contamination by disc stars. The final sample contains 17 objects previously described as star clusters in open cluster catalogues and a new one (Object 1), which was found on a sky survey plate by one of us (E. B.). Based on $\mathrm{DSS}^{3}$ and 2MASS data we determined accurate equatorial coordinates for the POCRs (Table 1).

Among the 18 sample objects, 8 were previously included in a list of 34 POCRs (Bica et al. 2001). Of these, 7 have been discussed in recent studies: (i) ESO 425 SC6 and ESO 425 SC15 were classified as non physical (Carraro et al. 2005); (ii) NGC 1663 was found to be a remnant (Baume et al. 2003); (iii) Ruprecht 3, NGC 1252 and NGC 1901 were found to be remnants (Pavani et al. 2003); (iv) NGC 6994 (Bassino et al. 2000; Carraro 2000; Ordenkirchen \& Soubiran 2002) is discussed further in the present work.

The intermediate-age open cluster NGC 3680 is used as a comparison (see also Bica et al. 2001). It presents evidence of an evolved open cluster dynamical stage and resembles in many respects what is expected for OCRs. The central luminosity function of NGC 3680 is nearly flat from the turnoff to the turnover (Bonatto et al. 2004). Depletion of the low MS in central parts and the presence of a corona rich in low-mass stars has been detected in NGC 3680 (see also Anthony-Twarog et al. 1989) The 2 MASS data are a powerful input in the analyses, making possible the study of (i) structural properties; (ii) objects and fields by means of a statistical method; (iii) the CMD distribution to derive ages, reddening, and distances.

The kinematical data were extracted from The Second US Naval Observatory CCD Astrograph Catalog - UCAC2 (Zacharias et al. 2004). It allows quantitative comparison of the proper motions of the object and large solid-angle offset fields. The use of complementary methods proved to be essential in the present study to deal with completeness effects affecting POCRs.

\subsection{Structural properties}

The 18 objects present two characteristic structures in the DSS/XDSS images. The first one is poorly populated with small angular sizes and is centrally concentrated. Such objects are hereafter referred to as compact (C in Table 1 ). The second structure type is more populated in stars, with larger angular sizes and more scattered stars. We refer to them as loose (L in Table 1).

NGC 3680 is similar to loose POCRs in the present sample (Fig. 2, in Bonatto et al. 2004). Figure 1 shows examples of $R$ band XDSS images of objects classified by eye as compact (left panels) and loose ones (right panels). The compact objects

3 The Digitized Sky Survey available: http://cadcwww.dao.nrc.ca/cadcbin/getdss 
Table 1. POCRs and open cluster NGC 3680.

\begin{tabular}{lcrrccc}
\hline \hline Name & $T$ & $\begin{array}{r}l \\
\left({ }^{\circ}\right)\end{array}$ & $\begin{array}{r}b \\
\left({ }^{\circ}\right)\end{array}$ & $\begin{array}{c}\alpha \\
(\mathrm{h}: \mathrm{m}: \mathrm{s})\end{array}$ & $\begin{array}{c}\delta \\
\left({ }^{\circ}::^{\prime \prime}\right)\end{array}$ & $\begin{array}{c}R_{\lim } \\
\left({ }^{\prime}\right)\end{array}$ \\
NGC 3680 & OC & 286.76 & 16.92 & $11: 25: 38$ & $-43: 14: 30$ & $22.0 \pm 1$ \\
\hline NGC 6481 & $\mathrm{C}$ & 29.94 & 14.93 & $17: 52: 48$ & $04: 10: 00$ & $1.5 \pm 0.5$ \\
M 73, NGC 6994 & $\mathrm{C}$ & 33.71 & -39.93 & $20: 58: 55$ & $-12: 38: 03$ & $4.5 \pm 0.5$ \\
NGC 6863 & $\mathrm{C}$ & 32.27 & -17.99 & $20: 05: 07$ & $-03: 33: 20$ & $1.5 \pm 0.5$ \\
NGC 1663, OCL-461 & $\mathrm{L}$ & 185.88 & -19.66 & $04: 49: 18$ & $13: 09: 47$ & $6.0 \pm 0.5$ \\
ESO 425 SC6 & $\mathrm{L}$ & 235.39 & -22.28 & $06: 04: 50$ & $-29: 10: 59$ & $3.0 \pm 0.5$ \\
ESO 425 SC15 & $\mathrm{L}$ & 236.37 & -20.35 & $06: 14: 35$ & $-29: 22: 30$ & $3.0 \pm 0.5$ \\
Ruprecht 3, OCL-642, ESO426SC33 & $\mathrm{C}$ & 238.77 & -14.81 & $06: 42: 07$ & $-29: 27: 15$ & $2.0 \pm 0.5$ \\
ESO 426 SC26 & $\mathrm{L}$ & 239.62 & -16.51 & $06: 36: 18$ & $-30: 51: 30$ & $3.0 \pm 0.5$ \\
ESO 429 SC2 & $\mathrm{L}$ & 242.59 & -4.16 & $07: 33: 24$ & $-28: 11: 15$ & $5.0 \pm 0.5$ \\
Ruprecht 31, OCL-711, ESO68SC9 & $\mathrm{C}$ & 250.08 & -5.97 & $07: 42: 58$ & $-35: 35: 50$ & $2.0 \pm 0.5$ \\
Waterloo 6 & $\mathrm{C}$ & 264.82 & -2.67 & $08: 40: 17$ & $-46: 07: 55$ & $1.5 \pm 0.5$ \\
ESO 570 SC12 & $\mathrm{L}$ & 274.06 & 35.89 & $11: 12: 07$ & $-21: 21: 00$ & $5.0 \pm 0.5$ \\
NGC 1252 & $\mathrm{L}$ & 274.08 & -50.83 & $03: 10: 49$ & $-57: 46: 00$ & $6.0 \pm 0.5$ \\
NGC 1901, Bok 1, OCL-791.1 & $\mathrm{L}$ & 279.03 & -33.60 & $05: 18: 11$ & $-68: 27: 00$ & $5.0 \pm 0.5$ \\
Object 1 & $\mathrm{C}$ & 294.94 & -0.63 & $11: 41: 34$ & $-62: 25: 05$ & $2.0 \pm 0.5$ \\
ESO 132 SC14 & $\mathrm{C}$ & 308.15 & 0.20 & $13: 36: 30$ & $-62: 12: 49$ & $1.5 \pm 0.5$ \\
ESO 383 SC10 & $\mathrm{L}$ & 312.13 & 27.08 & $13: 31: 30$ & $-35: 03: 56$ & $3.0 \pm 0.5$ \\
Lyngå 8, OCl-962, ESO 226SC1 & $\mathrm{C}$ & 333.27 & -0.07 & $16: 20: 04$ & $-50: 13: 59$ & $1.5 \pm 0.5$ \\
\hline
\end{tabular}

present a high-density contrast of stars as compared to the background and typically include up to 10 stars. Table 1 shows the 18 objects of the present sample, together with NGC 3680. The columns give: (1) designation; (2) structural type ( $\mathrm{C}$ for compact and L for loose); (3) and (4) Galactic longitude and latitude, respectively; (5) and (6) J2000.0 equatorial coordinates; (7) limiting radius $R_{\text {lim }}$ obtained by means of the radial stellar density profile (Figs. 2 and 3).

The occurrence of two distinct structures might be associated to different evolutionary phases and dynamical processes leading to a depletion of stars. Another possibility would be a different origin for compact and loose objects related, e.g., to formation conditions and position in the Galaxy.

The 2MASS Point Source Catalogue completeness limit ${ }^{4}$ is $99 \%$ for $J<15.8$ and $H<14.1$ at $|b|>30^{\circ}$. Basically, areas of incompleteness are at $\pm 75^{\circ}$ from the Galactic center, $b= \pm 1^{\circ}$ from the Galactic plane, and radius $r<5^{\circ}$ of the Galactic center. For these regions the completess limits are $J<14.0$ and $H<13.5 \mathrm{mag}$. Figures 7 to 9 of the 2MASS analysis of Release Catalogs 5 allow estimation of completess as a function of 1 and $b$. For the POCRs in Table 1 with $|b|>14^{\circ}$, the derived limits are $J<15.8$ and $H<14.1 \mathrm{mag}$, and for those with $|b|<6^{\circ} J<14.0$ and $H<13.5$ mag. Object 1, ESO132 SC14, and Lyngå 8 are close to the plane, and the CMD contains very red stars, so we applied a color filter (Sects. 4 and 5) to eliminate them, as well as to eliminate the contamination of LMC stars in NGC 1901 direction (see Fig. 9). Figures 2 and 3 show the radial density profiles for the magnitude-limited extractions for the whole sample.

The radial stellar density profile provides a general view of an open cluster structure. We apply this method to POCRs. Star counts with 2MASS photometry are carried out in concentric rings to take POCR and field areas into account. The extractions were centered in the equatorial coordinates of Table 1 . The radial density profiles were obtained from star counts in concentric rings with steps in radius depending on the object properties, divided by the ring area. The background contribution level shown

\footnotetext{
${ }^{4}$ http://www.ipac.caltech.edu/2mass/releases/allsky/ doc/sec6_5a1.html

${ }^{5}$ http://www.ipac.caltech.edu/2mass/releases/allsky/ doc/sec6.html
}

in Figs. 2 and 3 as shaded rectangles corresponds to the average number of stars present in an outer ring. Poisson error bars are significant since POCRs are intrinsically poorly populated.

As comparison, Fig. 4 of Bonatto et al. (2004) shows that the radial profile of the evolved open cluster NGC 3680 is described by a King model with core radius $r_{\mathrm{c}}=2.3 \pm 0.41^{\prime}$ and a limiting radius $R_{\text {lim }}=22 \pm 1^{\prime}$. Objects in Figs. 2 and 3 illustrate behaviors in the present sample. Compact POCRs as a rule show only one point above the background density. It basically corresponds to diameters estimated on DSS and XDSS images. Halos are generally absent. King models could not be fitted to the profiles of most POCRs. Loose POCRs present a density profile with an extended region (possibly a halo) characterizing their limiting radius, as illustrated by NGC 1663 (Fig. 2). The limiting radius arises where an observed profile merges with the background. We show the resulting limiting radii in Table 1. The loose POCRs NGC 1663, ESO 425 SC6, ESO 425 SC15, ESO 429 SC2, ESO 383 SC10 and NGC 1901 could be basically fitted by a King model. A comparison with the evolved open cluster NGC 3680 (Bonatto et al. 2004) shows that Poisson errors are larger for POCRs. It is important to explore loose POCRs as possibly intermediate evolutionary dynamical stages between open clusters and OCRs. Despite the loose appearance of ESO 570 SC12 (Fig. 1), its profile resembles those of compact POCRs, which might also suggest an evolutionary transition.

We conclude that the radial stellar density profiles of the sample objects are basically consistent with OCRs that gradually lose stars as compared to open clusters, persisting in the more internal parts responsible for observed limiting radii.

\section{3. $R^{2}$ statistical test}

Automated procedures for a systematic search of star clusters in catalogues have been developed in the past years. Reyle \& Robin (2001) combine the density of stars and the integrated flux in the $K_{\mathrm{s}}$ band. Ivanov et al. (2002) employed the apparent stellar surface density, $K_{\mathrm{s}}$ band luminosity function and the distribution of stars in $J-K_{\mathrm{s}}$. Mercer et al. (2005) present an algorithm that uses statistical methods to locate high stellar densities aided by criteria of color and magnitude selections. In general the main 

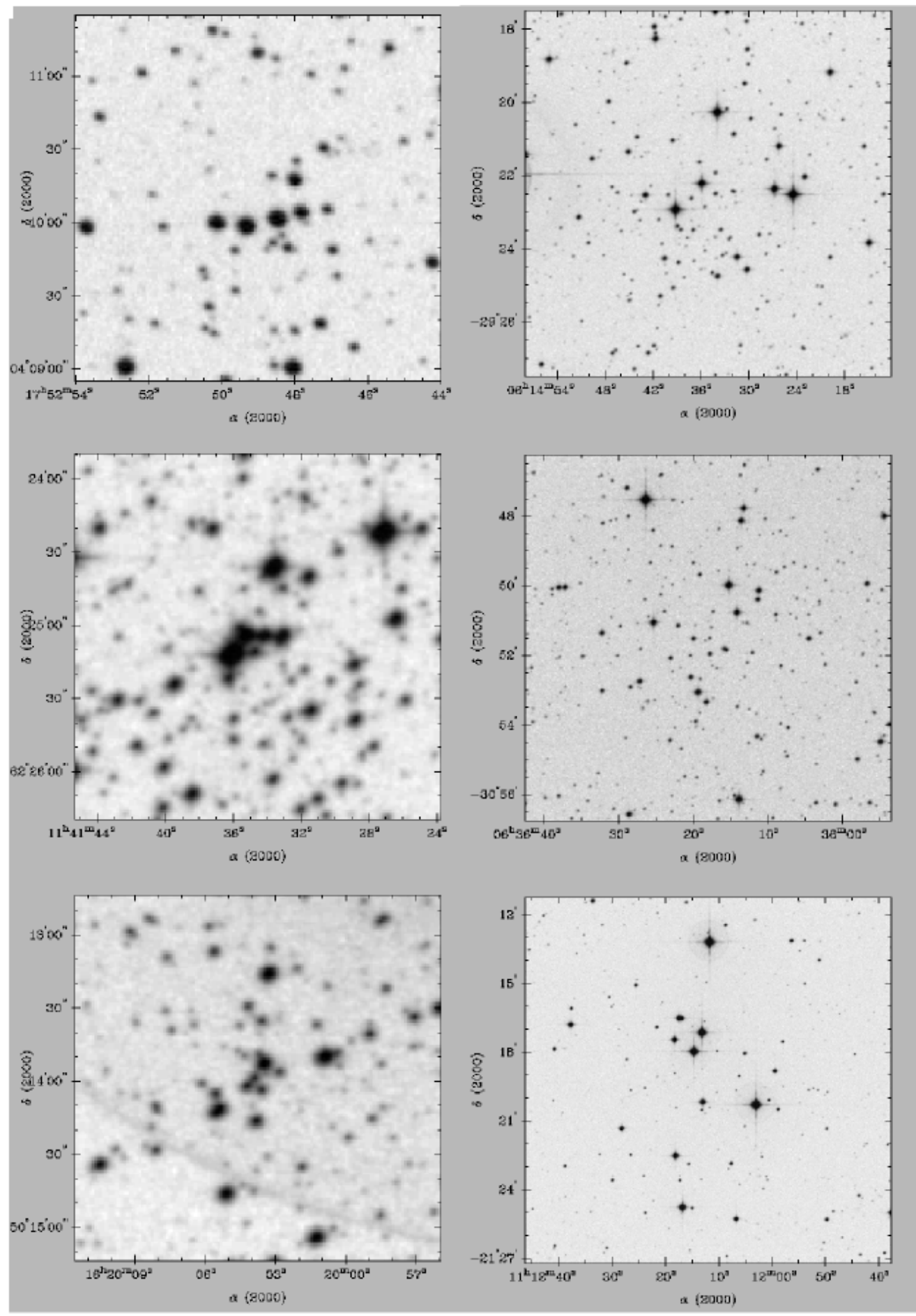

Fig. 1. XDSS R images for 6 sample objects. Left panels: compact objects NGC 6481 (top), Object 1 (middle), and Lyngå 8 (bottom). Right panels: loose objects (downwards) ESO 425 SC15, ESO 426 SC26, and ESO 570 SC12. North is to the top and east to the left.

objective of those searches is to reveal any hidden globular clusters, infrared star clusters, and young star clusters embedded in HII regions in our Galaxy. For known star clusters, there are e.g. studies from Mighell et al. (1996) and Kerber \& Santiago (2005) that applied statistical methods to compare populated clusters and fields using the CMD.

Although OCRs are less populated than open clusters, they should still present a stellar density contrast with respect to the surrounding field (Sect. 2.2). However, it is necessary to adapt the open cluster comparison methods owing to the intrinsically poorly populated nature of OCRs. Bica et al. (2001) compared the density of the bright stars in the POCR area with what is predicted for the field from a model of Galactic structure and $\mathrm{GSC}^{6}$ (Lasker et al. 1988) counts in an offset equal area.

We further develop and adapt statistical comparison methods to the 2MASS data of POCRs and test the hypothesis that the object can be reproduced in radial stellar density and CMD distribution by an equal-area field fluctuation. To perform this we

\footnotetext{
${ }^{6}$ The HST Guide Star Catalog, Version 1.2, available at http://www-gsss.stsci.edu/
} 
D. B. Pavani and E. Bica: Characterization of open cluster remnants
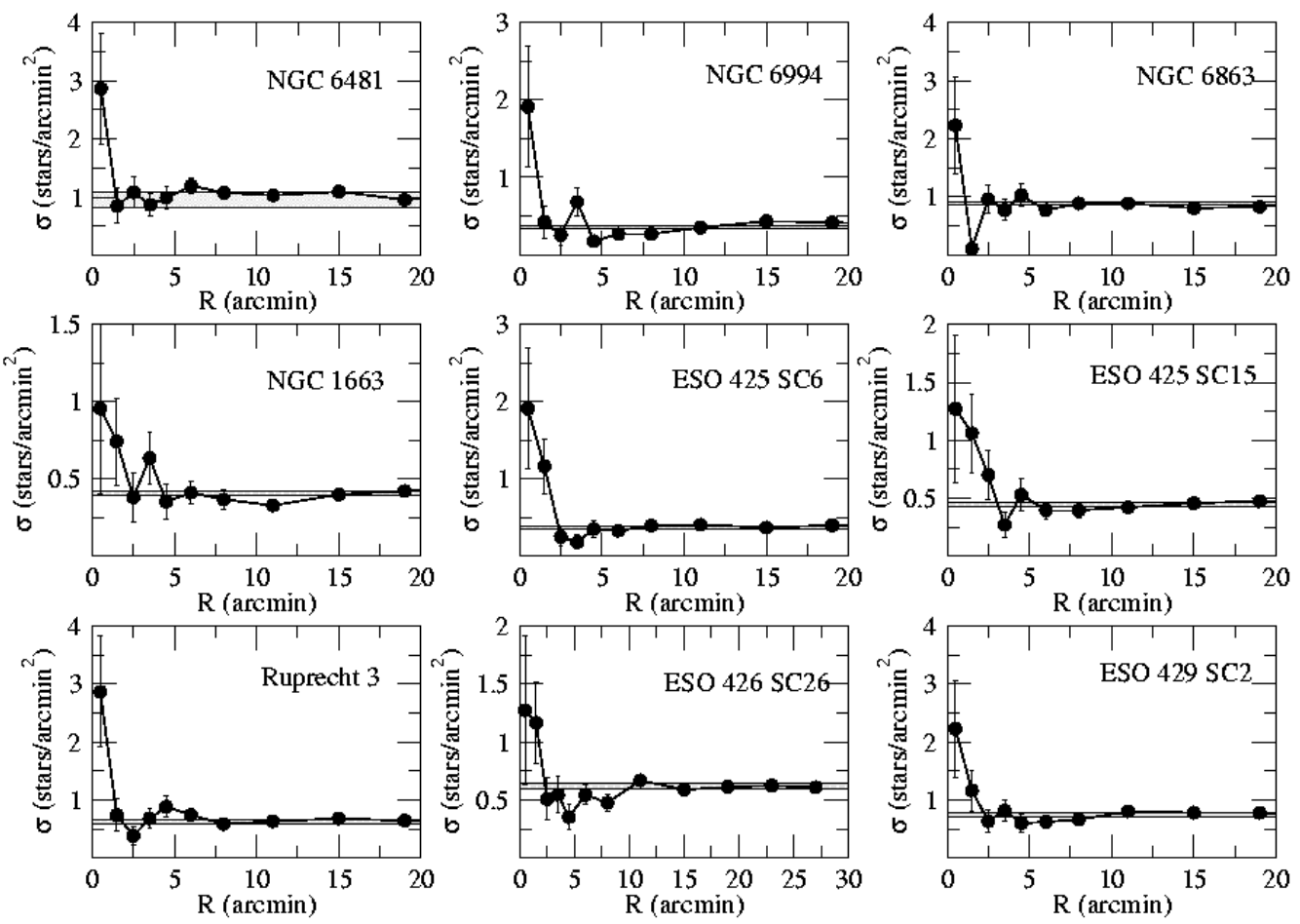

Fig. 2. Radial stellar density profile of sampled POCR. The average background level is shown (shaded rectangle). Poisson errors are also shown.
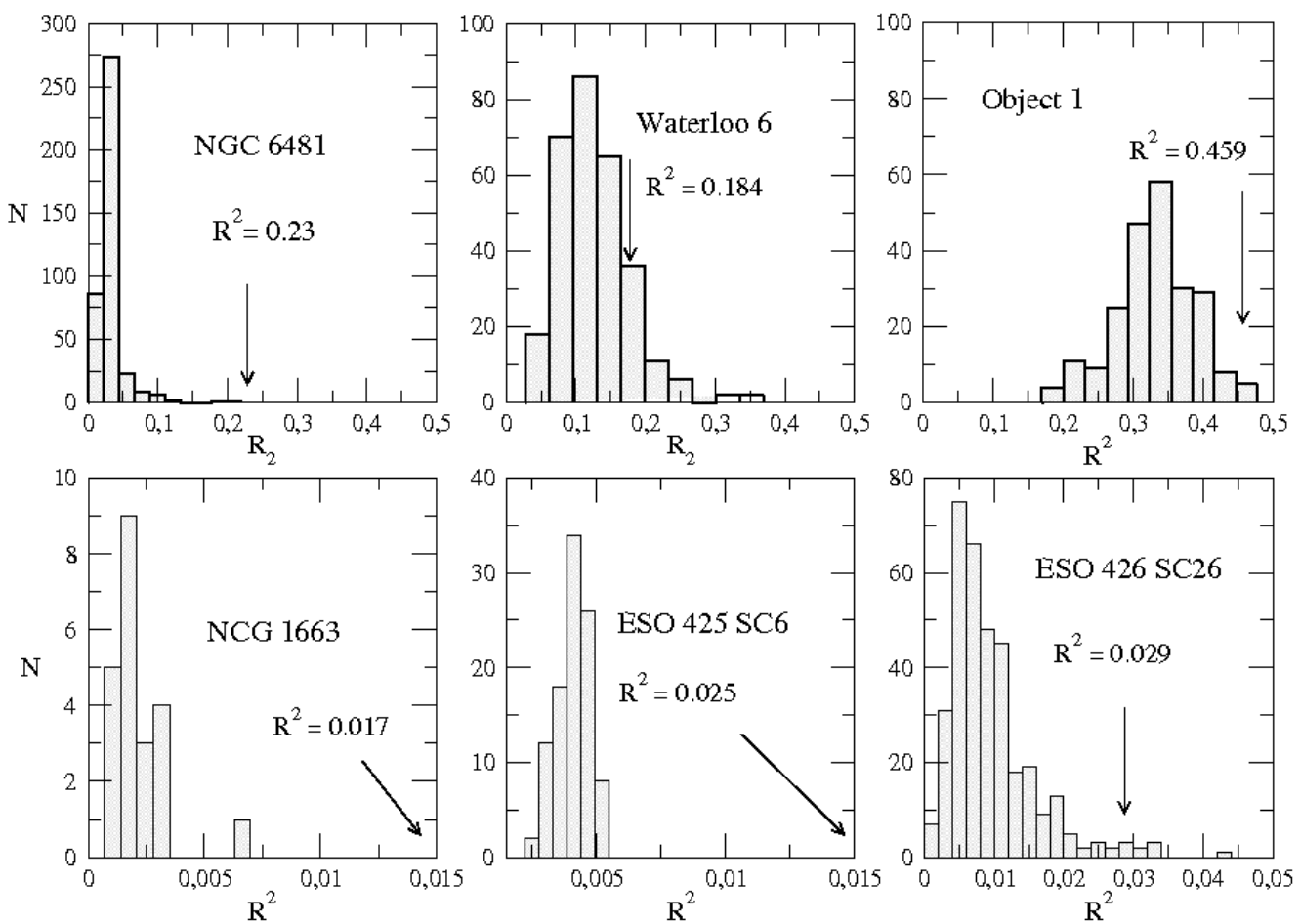

Fig. 4. Examples of $R^{2}$ statistical method testing how similar CMDs of objects and respective fields are. Histograms of representative field regions within the $R^{2}$ interval are given. The arrow shows the object locus. Top panels: NGC 6481 and Object 1 are examples of compact objects and appear to be physical ones, while Waterloo 6 appears to be a field fluctuation. Bottom panels: NGC 1663, ESO 425 SC6, ESO 426 SC26 are examples of loose objects that appear to be physical ones.

employ a nearby large-area offset field, with typical angular radius of 30' and separated from the object by $R_{\text {lim }}+3 \times R_{\text {lim }}$. Typically more than 100 sampling field areas of size equal to the $R_{\text {lim }}$ of each object (Table 1) were generated. Each CMD was divided into boxes with dimensions in color $\Delta(J-H)=0.125$ and magnitude $\Delta J=0.5 \mathrm{mag}$, generating a grid. We considered boxes containing at least one star. This grid was applied to the object and all samplings in the offset field. In each CMD box, the number of stars was counted and stellar densities were calculated using the object area. 
Table 2. Statistical test for POCRs and the open cluster NGC 3680.

\begin{tabular}{lrr}
\hline \hline Name & $\begin{array}{r}R_{\text {POCR }}^{2} \\
\left({ }^{\prime}\right)\end{array}$ & $\begin{array}{r}P \\
(\%)\end{array}$ \\
\hline NGC 3680 & 0.071 & $<1$ \\
\hline NGC 6481 & 0.232 & $<1$ \\
NGC 6994 & 0.006 & $<22$ \\
NGC 6863 & 0.104 & $<11.5$ \\
NGC 1663 & 0.017 & $<1$ \\
ESO 425 SC6 & 0.025 & $<1$ \\
ESO 425 SC15 & 0.027 & $<1$ \\
Ruprecht 3 & 0.077 & $<1$ \\
ESO 426 SC26 & 0.029 & $<6$ \\
ESO 429 SC2 & 0.025 & $<83$ \\
Ruprecht 31 & 0.197 & $<1.5$ \\
Waterloo 6 & 0.184 & $<20$ \\
ESO 570 SC12 & 0.005 & $<1$ \\
NGC 1252 & 0.001 & $<1$ \\
NGC 1901 & 0.030 & $<1$ \\
Object 1 & 0.459 & $<1.7$ \\
ESO 132 SC14 & 0.380 & $<1$ \\
ESO 383 SC10 & 0.024 & $<1$ \\
Lyngå 8 & 0.018 & $<1$ \\
\hline
\end{tabular}

Based on the $s^{2}$ (Kerber et al. 2001), we defined the $R^{2}$ statistics, which reflects the distribution of field fluctuations and density contrasts in the CMD plane between field and POCRs:

$R^{2}=\sum_{i=1}^{n_{\text {box }}}\left(\rho_{i}-\rho_{i, \text { field }}\right)^{2}$

where $n_{\text {box }}$ is the number of boxes in each grid for POCR (or sampled field CMD), $\rho_{i}$ corresponds to the box density for each

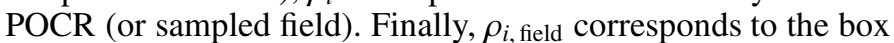
density for the total field.

The ensemble of $R^{2}$ for the sampled fields (e.g. Fig. 4) is a measure of the distribution of stars in the CMD of the total field, which in turn can be compared to that of the POCR. The method proved to be efficient for minimizing undersampling, which would otherwise be important if we had adopted the classical comparison between a populous open cluster and equal-area offset field.

Let $j_{\mathrm{POCR}}$ be the position of the POCR $R^{2}$ value $\left(R_{\mathrm{POCR}}^{2}\right)$ on the $R^{2}$ distribution for $N_{\mathrm{s}}$ sampled fields; we then define the probability $P$ of a POCR being a field fluctuation as:

$P=1-\frac{\left|j_{\mathrm{m}}-j_{\mathrm{POCR}}\right|}{N_{\mathrm{s}} / 2}$

where $j_{\mathrm{m}}$ is the median position in that distribution. Table 2 gives the $P$ and $R_{\text {POCR }}^{2}$ values for the POCRs. If the $R_{\mathrm{POCR}}^{2}$ value falls outside the $R^{2}$ field distribution, we adopt $P<1 \%$. NGC 6994, Waterloo 6, and ESO 429 SC2 attain $P<22 \%, P<20 \%$, and $P<83 \%$, respectively, and appear to be field fluctuations. NGC 6863 presents $P<11.5 \%$, and the remaining POCRs in Table 2 have very low $P$ values, favoring physical systems.

\section{CMD analyses}

We built 2MASS CMDs of POCRs and offset fields with equal extraction radii, corresponding to the region above the background field contributions in radial stellar density profile (Figs. 2 and 3). We applied a cutoff at $J=15$ and excluded sources with flags for non-stellar objects for all CMDs in order to minimize the star field contamination in POCR directions. We derived reddening, distance modulus, distance to the
Sun, Galactocentric distance, and age (Table 3) with the help of solar-metallicity Padova isochrones. In Fig. 5 we present the open cluster NGC 3680 core and off-core CMDs. The core CMD (left panel) presents a star distribution that is expected for OCRs: depletion of the low MS in central parts and the presence of a corona rich in low-mass stars. The field of NGC 3680 at $b \sim$ $17^{\circ}$ is uncrowded according to the 2 MASS $99 \%$ completeness limit. NGC 3680 is probably not affected by severe crowding, since the brighter limit found in $2 \mathrm{MASS}$ crowded fields ${ }^{7}$ occurs only near the plane or central parts of globular clusters. The central MS depletion (Fig. 5) was also observed by Anthony-Twarog et al. (1989). Since POCRs are less populous than open clusters, incompleteness is not expected. The brighter stars in the images, which in general characterize a POCR (Fig. 1), were identified in the CMD, which provided constraints for isochrone fittings.

The NGC 1901 direction is contaminated by LMC stars. We eliminated them by means of the color filters shown in Fig. 9. To verify contamination in the NGC 1901 direction we analyzed three CMDs in three filters of fields with the same angular size as that of NGC 1901: (i) the field of the globular cluster NGC 1928 projected near the center of the LMC bar; (ii) the field of the association of stars NGC 2055 near the east end of the LMC bar dominated by a young population; and (iii) the field of NGC 2004 with red supergiants. Figures 7 and 9 dotted lines show the residual LMC contamination in NGC 1901 and offset field directions, respectively. Several POCRs have tight MS distributions, such as NGC 6481, ESO 425 SC6, and ESO 426 SC26 (Fig. 6). A sequence of binaries, together with that of single stars in general, encompass most of the POCR stars. The sequence of binaries is obtained from that of single stars by a shift of $\triangle J=0.75 \mathrm{mag}$ (Santiago et al. 1996; Elson et al. 1998). As a rule, offset fields are underpopulated and, scattered and stars do not show the same brightness range (Figs. 8 and 9). On the other hand, in the CMDs of Waterloo 6 and ESO 132 SC14 (Fig. 7) stars are more scattered.

We applied a numerical method to quantify the fit of an isochrone to a POCR CMD. Stars compatible with single and binary isochrones were counted taking error bars into account. We obtained their ratio to the total number of stars in the CMD, defined as the fitting index $I_{\mathrm{f}}$. Best isochrone fits could be obtained for all objects, and tentative fundamental parameters were derived (Table 3). This suggests that only classical CMD analyses may not be enough for conclusive results on the nature of underpopulated objects. The ensemble of methods will settle the more probable physical objects (Sect. 6). Table 3 gives (1) designation, (2) the extraction radius of the data, (3) $I_{\mathrm{f}}$, (4) age $\tau$ (Gyr), (5) near IR and (6) optical reddening values, (7) distance modulus, (8) distance to the Sun $d_{\odot}(\mathrm{kpc}),(9)$ linear diameter (pc), (10) height from the plane $z(\mathrm{kpc}),(11)$ Galactocentric distance $R_{\mathrm{gc}}(\mathrm{kpc})$, and (12) number of probable members.

Linear diameters were are calculated from angular limiting radii (Table 1) and distances to the Sun. While most POCRs have diameters in the range 1-2 pc (Table 3 ), open clusters such as M 67 and NGC 3680, at comparable ages and Galactocentric distances, have 17.4 and $12.8 \mathrm{pc}$, respectively (Bonatto et al. 2004). This suggests that POCRs correspond to open cluster cores. The $E(B-V)$ values were coverted from $E(J-H)$ using $E(B-V)=3.03 \times E(J-H)($ Rieke \& Lebofsky 1985). The number of probable members was estimated from $I_{\mathrm{f}}$ as was the total number of stars in the POCR extraction radius. The number of probable members was not estimated for POCRS with $I_{\mathrm{f}}<50 \%$.

\footnotetext{
7 http://www .ipac. caltech.edu/2mass/releases/allsky/ doc/sec6.html
} 
Table 3. CMDs parameters for POCRs and the open cluster NGC 3680.

\begin{tabular}{|c|c|c|c|c|c|c|c|c|c|c|c|}
\hline Name & $\begin{array}{l}r_{\mathrm{d}} \\
\left({ }^{\prime}\right) \\
\end{array}$ & $\begin{array}{c}I_{\mathrm{f}}(\%) \\
(\%)\end{array}$ & $\begin{array}{c}\tau(\mathrm{Gyr}) \\
(\mathrm{Gyr})\end{array}$ & $\begin{array}{c}E(J-H) \\
(\mathrm{mag})\end{array}$ & $\begin{array}{c}E(B-V) \\
(\mathrm{mag})\end{array}$ & $\begin{array}{c}\left(J-M_{J}\right) \\
(\mathrm{mag})\end{array}$ & $\begin{array}{c}d_{\odot} \\
(\mathrm{kpc})\end{array}$ & $\begin{array}{c}D \\
(\mathrm{pc})\end{array}$ & $\begin{array}{c}z \\
(\mathrm{kpc})\end{array}$ & $\begin{array}{c}R_{\mathrm{gc}} \\
(\mathrm{kpc})\end{array}$ & $\mathrm{pm}$ \\
\hline NGC 3680 & 3.0 & $78 \pm 18$ & $1.6 \pm 0.01^{a}$ & $0.0^{a}$ & 0.0 & $10.00^{a}$ & $1.00^{a}$ & $12.8^{a}$ & 0.30 & $8.8^{a}$ & 18 \\
\hline NGC 6481 & 1.5 & $83 \pm 26$ & $3.5 \pm 0.5$ & 0.06 & 0.18 & 10.36 & 1.18 & 1.03 & 0.30 & 7.04 & 10 \\
\hline NGC 6994 & 2.5 & $83 \pm 26$ & $1.0 \pm 0.2$ & 0.01 & 0.03 & 9.77 & 0.9 & 2.36 & -0.57 & 8.0 & 10 \\
\hline NGC 6863 & 1.5 & $75 \pm 31$ & $3.5 \pm 0.5$ & 0.00 & 0.00 & 10.40 & 1.20 & 1.05 & -0.37 & 7.06 & 7 \\
\hline NGC 1663 & 3.0 & $88 \pm 23$ & $2.8 \pm 0.2$ & 0.04 & 0.12 & 0.20 & 1.10 & 4.20 & -0.40 & 9.13 & 15 \\
\hline ESO 425 SC6 & 2.5 & $71 \pm 18$ & $2.5 \pm 0.3$ & 0.00 & 0.00 & 10.20 & 1.10 & 1.92 & -0.41 & 8.62 & 15 \\
\hline ESO $425 \mathrm{SC} 15$ & 3.0 & $61 \pm 15$ & $1.0 \pm 0.3$ & 0.01 & 0.03 & 9.97 & 0.99 & 1.73 & -0.34 & 8.55 & 15 \\
\hline Ruprecht 3 & 2.0 & $65 \pm 18$ & $1.5 \pm 0.5$ & 0.00 & 0.00 & 10.20 & 1.10 & 1.28 & -0.28 & 8.60 & 13 \\
\hline ESO 426 SC26 & 2.5 & $70 \pm 17$ & $1.0 \pm 0.3$ & 0.01 & 0.04 & 10.46 & 1.24 & 2.16 & -0.35 & 8.66 & 17 \\
\hline ESO 429 SC2 & 1.5 & $68 \pm 17$ & $0.4 \pm 0.05$ & 0.03 & 0.9 & 11.20 & 1.67 & 4.85 & -0.12 & 8.88 & 15 \\
\hline Ruprecht 31 & 1.5 & $78 \pm 24$ & $1.0 \pm 0.3$ & 0.02 & 0.06 & 9.85 & 0.93 & 0.81 & -0.10 & 8.36 & 11 \\
\hline Waterloo 6 & 1.5 & $48 \pm 15$ & $0.2 \pm 0.1$ & 0.00 & 0.00 & 10.5 & 1.26 & 1.28 & -0.06 & 8.21 & - \\
\hline ESO 570 SC 12 & 4.0 & $62 \pm 20$ & $0.8 \pm 0.2$ & 0.01 & 0.03 & 9.02 & 0.64 & 1.86 & 0.37 & 7.99 & 10 \\
\hline NGC 1252 & 4.0 & $65 \pm 19$ & $2.8 \pm 0.3$ & 0.00 & 0.00 & 9.5 & 0.79 & 2.76 & -0.61 & 8.00 & 11 \\
\hline NGC 1901 & 5.0 & $67 \pm 21$ & $0.6 \pm 0.1$ & 0.01 & 0.03 & 8.35 & 0.46 & 1.34 & -0.26 & 7.95 & 10 \\
\hline Object 1 & 1.5 & $50 \pm 11$ & $0.6 \pm 0.1$ & 0.00 & 0.00 & 11.20 & 1.74 & 2.00 & -0.02 & 7.44 & 20 \\
\hline ESO 132 SC14 & 1.5 & $33 \pm 9$ & $0.8 \pm 0.2$ & 0.00 & 0.00 & 10.20 & 1.10 & 0.95 & 0.003 & 7.37 & - \\
\hline ESO 383 SC10 & 3.0 & $76 \pm 19$ & $2.0 \pm 0.5$ & 0.00 & 0.00 & 10.00 & 1.00 & 1.74 & 0.45 & 7.45 & 15 \\
\hline Lyngå 8 & 1.5 & $56 \pm 19$ & $2.0 \pm 0.5$ & 0.19 & 0.57 & 10.11 & 1.05 & 0.92 & -0.001 & 7.07 & 9 \\
\hline
\end{tabular}

${ }^{a}$ Bonatto et al. (2004).

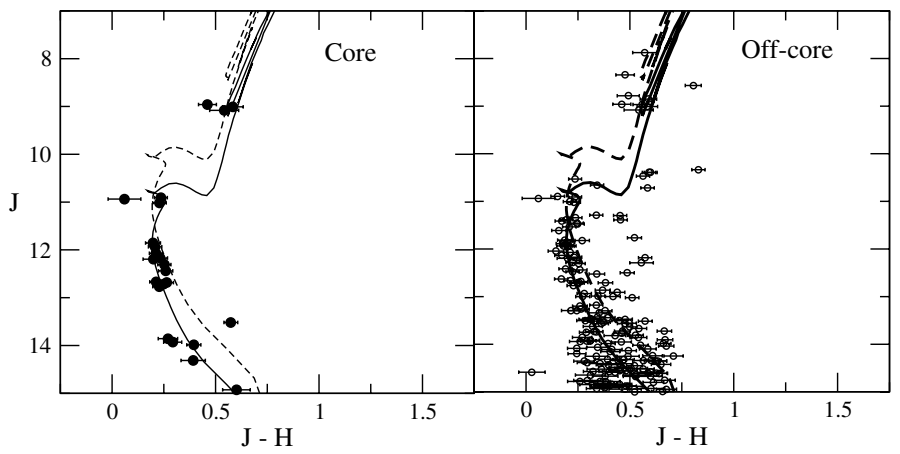

Fig. 5. $J \times(J-H)$ CMD of NGC 3680 in core (left panel) and offcore (right panel) regions. Padova isochrones with solar metallicity and shifted ones according to binarity (dashed line) are shown. Photometric errors are indicated.

Typical errors are $\epsilon(E(J-H))=0.02 \mathrm{mag}, \epsilon\left(J-M_{J}\right)=0.2 \mathrm{mag}$, $\epsilon\left(d_{\odot}\right)=0.1 \mathrm{kpc}, \epsilon(z)=0.015 \mathrm{kpc}, \epsilon\left(R_{\mathrm{gc}}\right)=0.4 \mathrm{kpc}$. The errors combine 2MASS photometric errors with CMD fit errors. Using the relation $A_{J} / A_{\mathrm{V}}=0.282$ (Rieke \& Lebofsky 1985), we derived $A_{J}=2.65 \times E(J-H)$ in the calculations above. In the sample 13 POCRs with $I_{\mathrm{f}}>60 \%$ are described by the respective isochrones well. Three POCRs have $50 \% \leq I_{\mathrm{f}} \leq 60 \%$, while Waterloo 6 and ESO 132 SC14 have $I_{\mathrm{f}}<50 \%$.

We determined ages by fitting isochrones to the extracted CMDs and using equal area fields as references. No decontamination procedure was necessary. Most sample POCRs are located at relatively high Galactic latitudes and thus are favored by low contamination. The 6 POCRs at $|b|<6^{\circ}$ have small angular diameters and bluer colors on average than the field stars (Fig. 7).

\section{Proper motions}

Bica \& Bonatto (2005) present internal proper motion distributions that are related to binary stars in open clusters. They show that proper motions are a useful tool for identifying highervelocity stars as unresolved binary cluster members and, as a consequence, for mapping and quantifying the binary fraction in the CMD.

Individual POCRs show low stellar statistics as compared to open clusters, and composite POCR CMDs might provide more insight into their nature. Proper motions extracted from UCAC2 allowed a comparison between POCR areas and large offset fields.

We applied a color filter (Bonatto et al. 2005) to the CMD of a given POCR and offset field stars that removed most background contamination. The color filter was used to separate the CMD of a POCR from background contamination (most disc and/or bulge stars), leaving a residual contamination that will be taken into account by means of histogram subtractions. In the following we employ the modulus of proper motion components

$$
M P=\sqrt{\left(\mu_{\alpha} \cos \delta\right)^{2}+\mu_{\delta}^{2}}
$$

To be consistent with the CMD analysis, we extracted the data for each POCR inside the limiting radius $R_{\text {lim }}$ (Table 1 ). We verified that the correspondence between UCAC2 and 2MASS is nearly complete for $J \leqslant 14.5$.

Histograms of stars in proper motion modulus bins of 2 mas/yr were built for POCRs with $I_{\mathrm{f}} \geqslant 50 \%$ and their offset fields. The offset field was scaled to the POCR area. We subtracted the offset field histogram from that of the POCR region, and the result provides the proper motion distribution in Figs. 10 and 11. Asymmetries and peaks in the proper motion distributions may yield information on the internal kinematics, presence of binaries, and possible evolutionary stages.

In order to analyze intrinsic properties, we transformed proper motions into linear velocities on the plane of the sky $\left(v_{\mathrm{p}}\right)$. The units mas/yr convert to $\mathrm{km} / \mathrm{s}$ using the distances in Table 3 and

$v_{\mathrm{p}}\left(\mathrm{km} \mathrm{s}^{-1}\right)=M P(\operatorname{mas} / \mathrm{yr}) * d(\mathrm{pc}) * 4.74 \times 10^{-3}$.

The objects were divided into two groups (A and $\mathrm{B}$ ) according to their $v_{\mathrm{p}}$ distributions characterized by peaks or flatter distributions. The observed median velocities $\left(v_{\mathrm{M}}\right)$ were derived for rest velocity corrections (Table 4), and the median is suitable for non Gaussian distributions. 


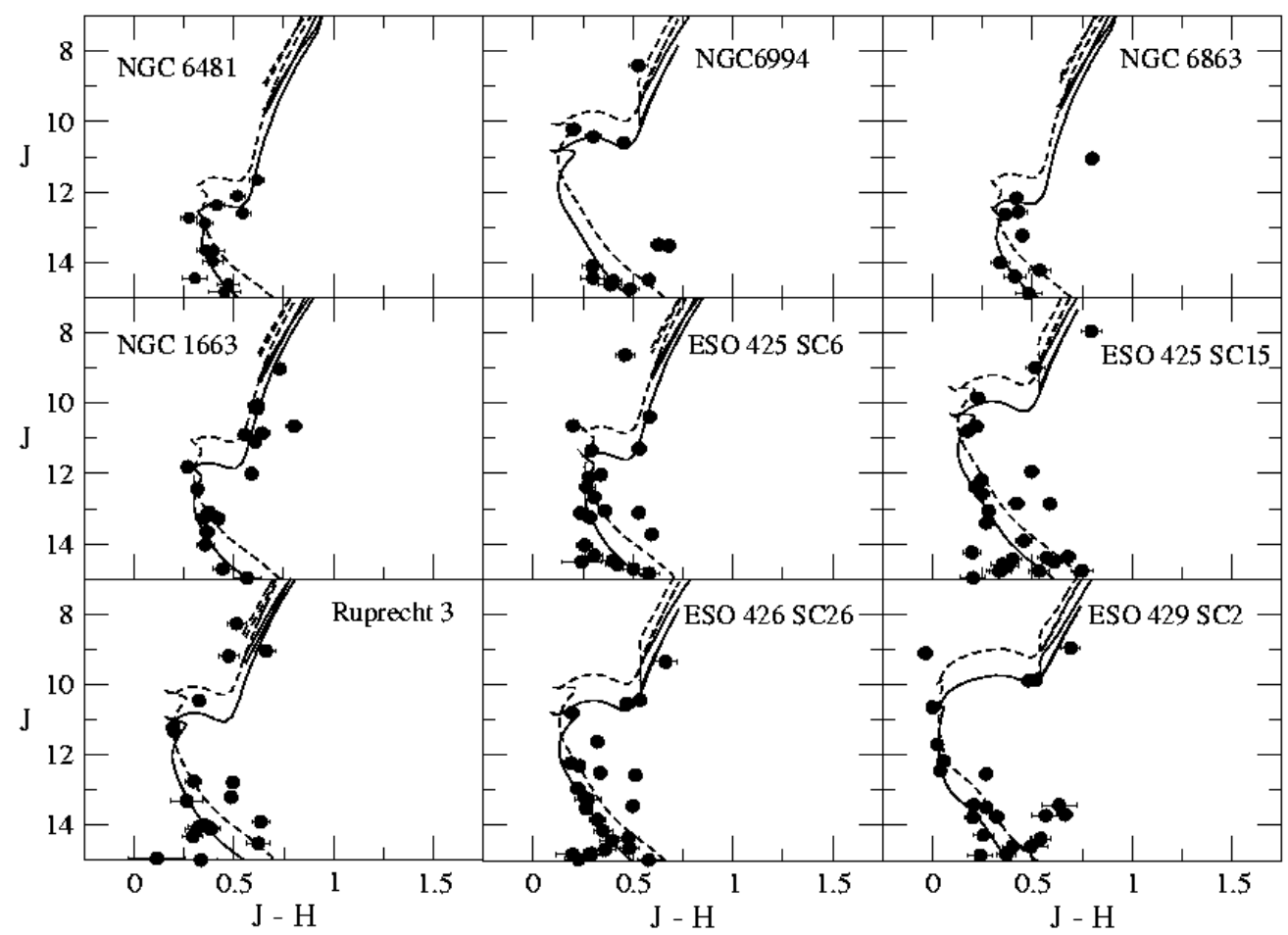

Fig. 6. $J \times(J-H)$ CMDs of POCRs. Padova isochrones with solar metallicity, and the same shifted according to binarity (dashed) are shown, and photometric errors indicated.

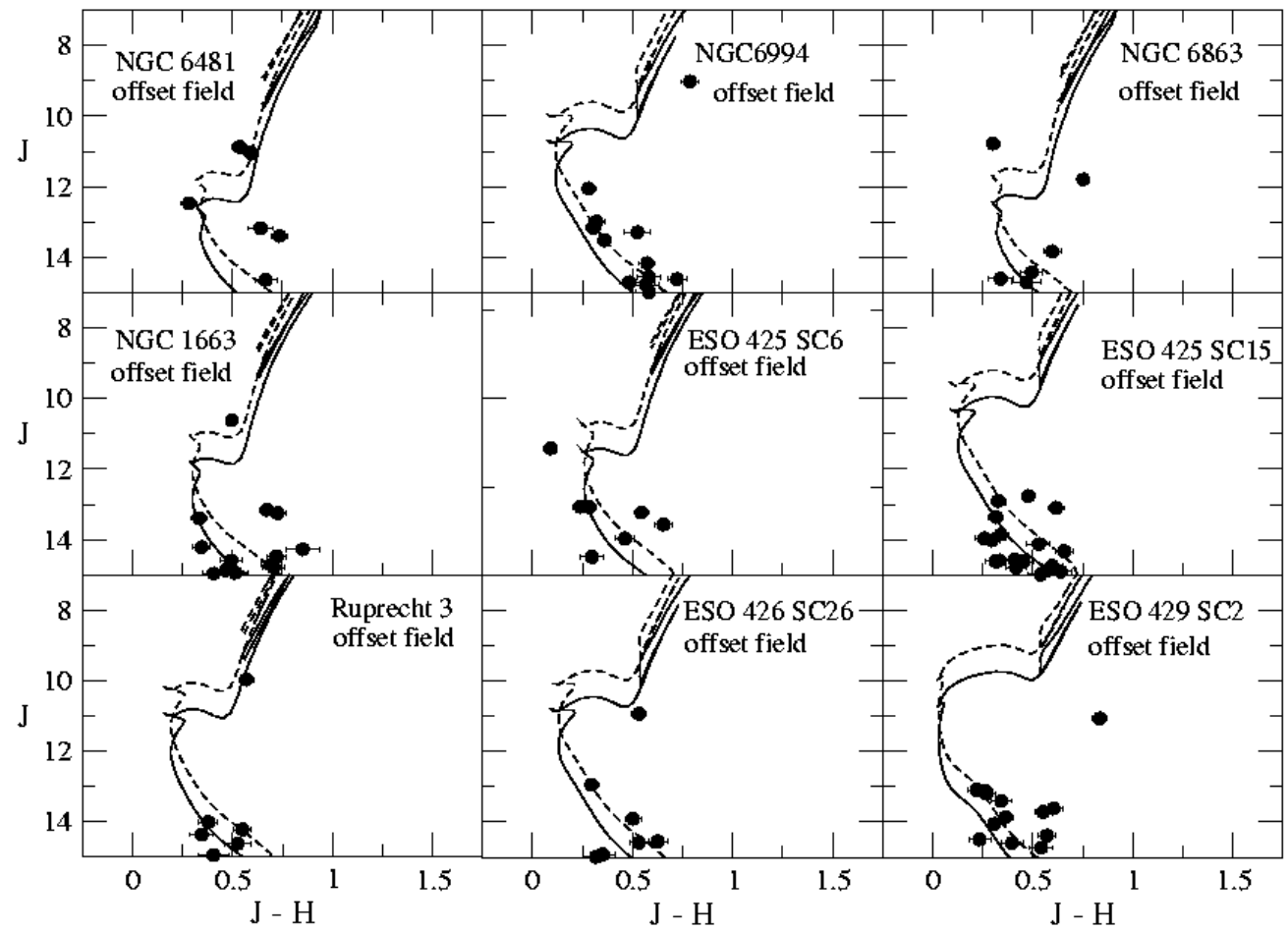

Fig. 8. Same as Fig. 6 for offset fields.

Finally, composite velocity histograms were built: (i) $v_{\mathrm{M}}$ in each POCR histogram was used to correct the diagram to rest velocity, providing it in the form $\left(v_{\mathrm{p}}-v_{\mathrm{M}}\right)$; (ii) Group A is characterized by a well-defined low-velocity peak and is formed by the loose POCRs NGC 1663, ESO 425 SC15, ESO 426 SC26, NGC 1252, NGC 1901, and the compact one Ruprecht 31. They have $\left(v_{\mathrm{M}}<71 \mathrm{~km} \mathrm{~s}^{-1}\right)$. Group B is characterized by highervelocities, $75 \leqslant v_{\mathrm{M}}<140 \mathrm{~km} \mathrm{~s}^{-1}$, and is formed by the compact objects NGC 6481, NGC 6863, Ruprecht 3, Object 1 , Lyngå 8 and the loose ones, ESO 425 SC6, ESO 570 SC12, and ESO 383 SC10.

Figure 12 presents the composite histograms $\left(v_{p}-v_{M}\right)$ for Groups A and B. Group A shows a well-defined low-velocity peak. Group B shows a double peak. Table 4 gives the POCRs belonging to each group. It is outstanding that the $\left(v_{\mathrm{p}}-v_{\mathrm{M}}\right)$ composite histogram reveals properties of POCRs only seen before 


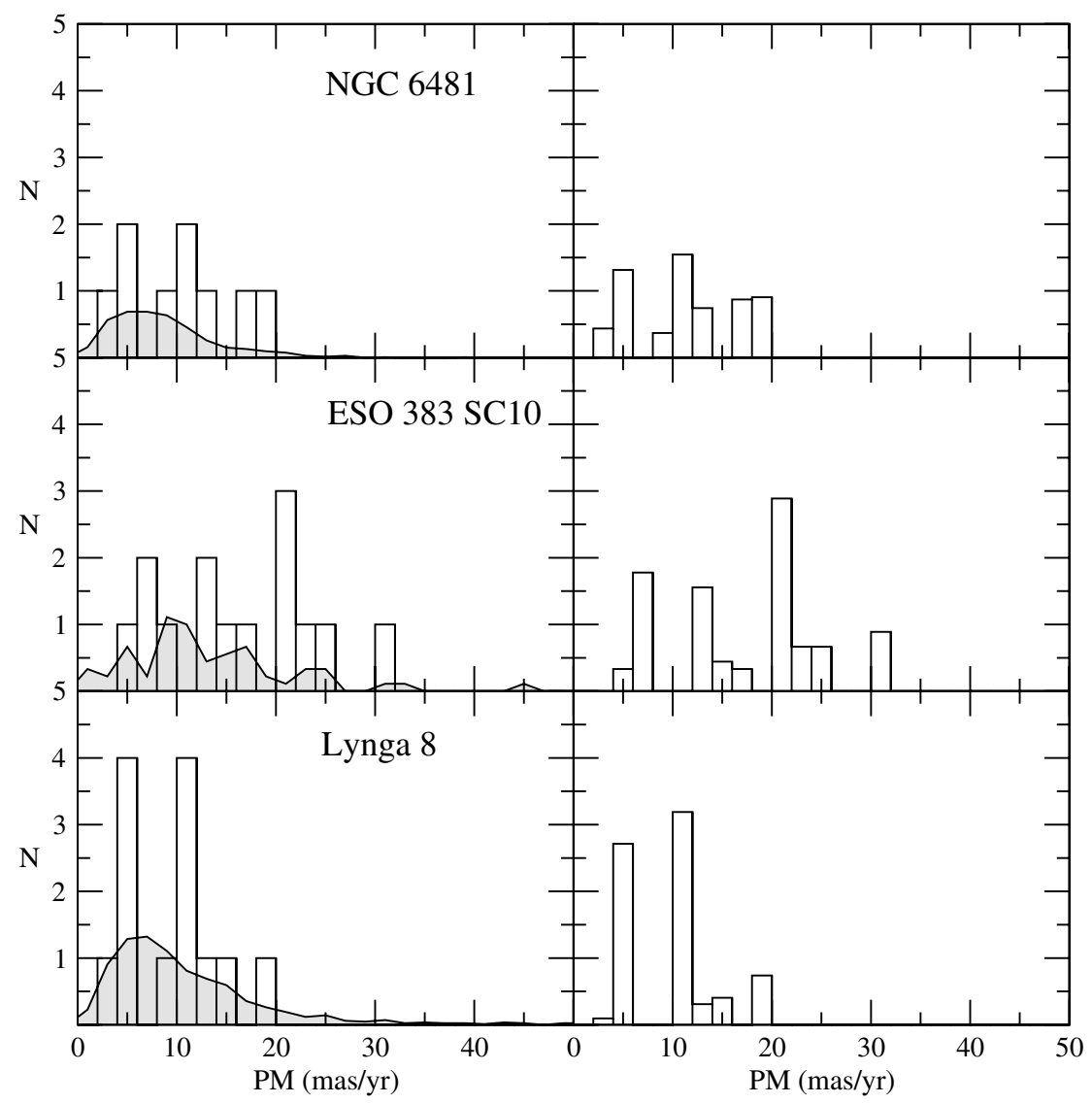

Fig. 11. Same as Fig. 10, for POCRs with a flatter distribution of the modulus of proper motion.

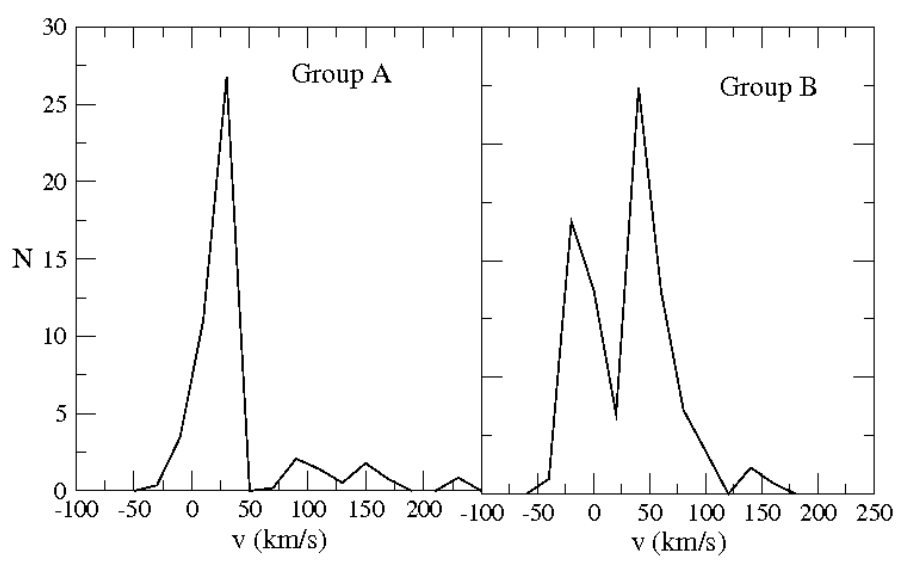

Fig. 12. Distribution of velocities projected on the sky reduced to the rest velocity. Left panel: Group A. Right panel: Group B. A velocity bin of $20 \mathrm{~km} \mathrm{~s}^{-1}$ was used.

in open clusters (Bica \& Bonatto 2005), which cannot be observed in individual POCRs owing to low statistics. The doublepeak distribution in Group B resembles that of single (low velocity) and unresolved binary stars (high velocity) as observed in off-core regions of M 67 (e.g. Fig. 5 in Bica \& Bonatto 2005).

Several objects were not included in the group analysis because of doubt about their nature or they are field fluctuations because the indices are indicating non physical objects (Table 5). Although ESO 429 SC2 has a proper motion distribution compatible with Group A and has $I_{\mathrm{f}}=68 \pm 16 \%$, it was not included in the composite histogram due to the probability of being a field fluctuation (Table 2). The CMD of NGC 6994 (Fig. 6)
Table 4. The groups and median velocity values.

\begin{tabular}{llr}
\hline \hline Name & Type & $v_{\mathrm{M}}\left(\mathrm{km} \mathrm{s}^{-1}\right)$ \\
\hline Group A & & \\
\hline NGC 1663 & $\mathrm{L}$ & 68 \\
ESO 425 SC15 & $\mathrm{L}$ & 47 \\
ESO 426 SC26 & $\mathrm{L}$ & 70 \\
Ruprecht 31 & $\mathrm{C}$ & 44 \\
NGC 1252 & $\mathrm{L}$ & 68 \\
NGC 1901 & $\mathrm{L}$ & 26 \\
\hline Group B & & \\
\hline NGC 6481 & $\mathrm{C}$ & 78 \\
NGC 6863 & $\mathrm{C}$ & 137 \\
ESO 425 SC6 & $\mathrm{L}$ & 83 \\
Ruprecht 3 & $\mathrm{C}$ & 83 \\
ESO 570 SC12 & $\mathrm{L}$ & 109 \\
Object 1 & $\mathrm{C}$ & 82 \\
ESO 383 SC10 & $\mathrm{L}$ & 113 \\
Lyngå 8 & $\mathrm{C}$ & 75 \\
\hline
\end{tabular}

shows a gap of $\approx 3$ mag. between bright and faint stars that is improbable in a turnoff (TO) to low MS distribution. The statistical method (Table 2) also favors a non physical nature. These results point to NGC 6994 being a field fluctuation, in agreement with Carraro (2005) and Odenkirchen \& Soubiran (2002).

Composite open cluster CMDs have been a tool employed since Sandage (1958) and were useful for modeling stellar evolution. In the present study we applied this principle to OCRs and POCRs. By means of reddening and distance corrections, we obtained absolute CMDs, which were in turn combined to build composite CMDs for Groups A and B, improving the stellar 


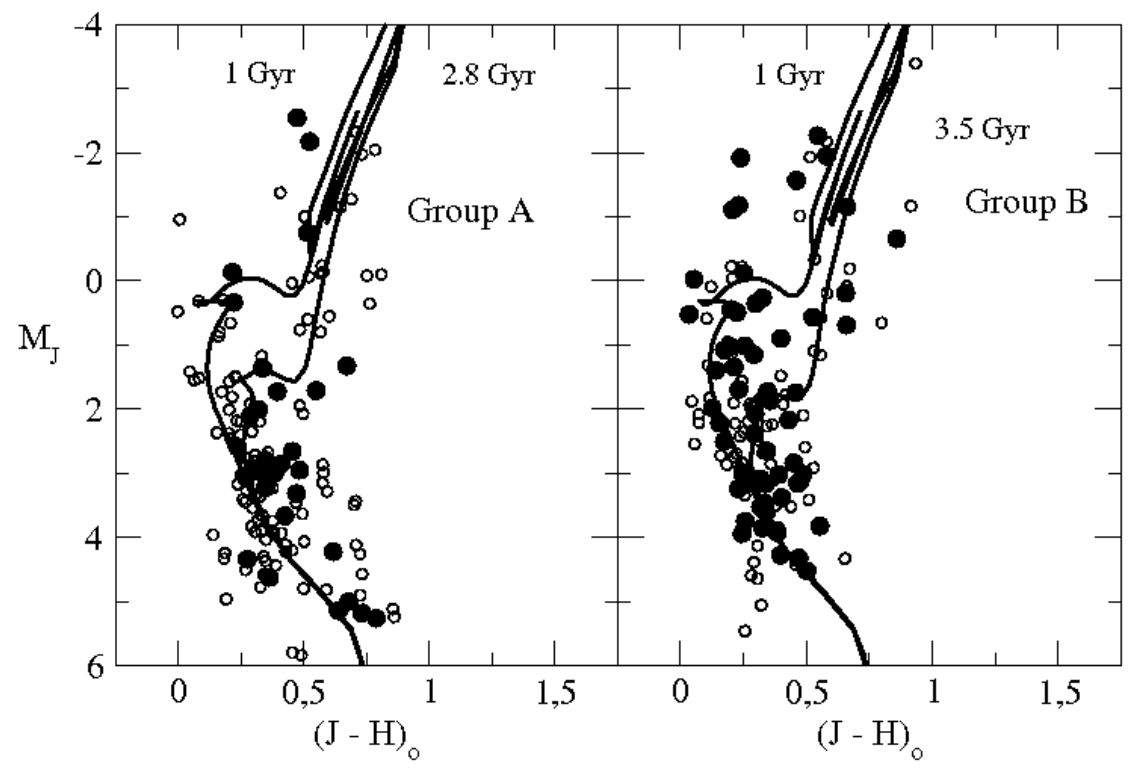

Fig. 13. Absolute CMDs for Groups A and B (left and right panels, respectively). Stars with velocities $\left(v_{\mathrm{p}}-v_{\mathrm{M}}\right)$ higher than $25 \mathrm{~km} \mathrm{~s}^{-1}$ (filled circles) are superimposed on the CMDs. Padova isochrones are shown as reference.

Table 5. Final classification for the POCRS.

\begin{tabular}{lccclc}
\hline \hline Nome & Type & Profile & $R^{2}$ & $I_{\mathrm{f}}$ & Q \\
\hline NGC 3680 & $\mathrm{L}$ & $\mathrm{y}$ & $\mathrm{y}$ & $\mathrm{y}$ & OC \\
\hline NGC 6481 & $\mathrm{C}$ & $\mathrm{y}$ & $\mathrm{y}$ & $\mathrm{y}$ & OCR \\
NGC 6994 & $\mathrm{C}$ & $\mathrm{y}$ & $\mathrm{n}$ & $\mathrm{n}$ & FF \\
NGC 6863 & $\mathrm{C}$ & $\mathrm{y}$ & $\mathrm{y}$ & $\mathrm{y}$ & OCR \\
NGC 1663 & $\mathrm{L}$ & $\mathrm{y}$ & $\mathrm{y}$ & $\mathrm{y}$ & OCR \\
ESO 425 SC 6 & $\mathrm{L}$ & $\mathrm{y}$ & $\mathrm{y}$ & $\mathrm{y}$ & OCR \\
ESO 425 SC 15 & $\mathrm{L}$ & $\mathrm{y}$ & $\mathrm{y}$ & $\mathrm{y}$ & OCR \\
Ruprecht 3 & $\mathrm{C}$ & $\mathrm{y}$ & $\mathrm{y}$ & $\mathrm{y}$ & OCR \\
ESO 426 SC 26 & $\mathrm{L}$ & $\mathrm{y}$ & $\mathrm{y}$ & $\mathrm{y}$ & OCR \\
ESO 429 SC 2 & $\mathrm{L}$ & $\mathrm{y}$ & $\mathrm{n}$ & $\mathrm{y}$ & POCR \\
Ruprecht 31 & $\mathrm{C}$ & $\mathrm{y}$ & $\mathrm{y}$ & $\mathrm{y}$ & OCR \\
Waterloo 6 & $\mathrm{C}$ & $\mathrm{y}$ & $\mathrm{n}$ & $\mathrm{n}$ & FF \\
ESO 570 SC 12 & $\mathrm{L}$ & $\mathrm{y}$ & $\mathrm{y}$ & $\mathrm{y}$ & OCR \\
NGC 1252 & $\mathrm{L}$ & $\mathrm{y}$ & $\mathrm{y}$ & $\mathrm{y}$ & OCR \\
NGC 1901 & $\mathrm{L}$ & $\mathrm{y}$ & $\mathrm{y}$ & $\mathrm{y}$ & OCR \\
Object 1 & $\mathrm{C}$ & $\mathrm{y}$ & $\mathrm{y}$ & $\mathrm{n}$ & POCR \\
ESO 132 SC 14 & $\mathrm{C}$ & $\mathrm{y}$ & $\mathrm{y}$ & $\mathrm{n}$ & POCR \\
ESO 383 SC 10 & $\mathrm{S}$ & $\mathrm{y}$ & $\mathrm{y}$ & $\mathrm{y}$ & OCR \\
Lyngå 8 & $\mathrm{C}$ & $\mathrm{y}$ & $\mathrm{y}$ & $\mathrm{y}$ & OCR \\
\hline & & & & &
\end{tabular}

statistics in the diagrams (Fig. 13). Considering the $\left(v_{\mathrm{p}}-v_{\mathrm{M}}\right)$ distributions for Groups A and B, stars with velocities higher than $25 \mathrm{~km} \mathrm{~s}^{-1}$ are more probably related to unresolved binary stars, based on analysis of the populous open cluster M67 (Bica \& Bonatto 2005). We plotted those stars in the composite CMD and they tend to be located to the right of the MS of single stars, as expected (Fig. 13).

Group A (Fig. 13) shows the low-velocity stars distributed along the MS for different ages, while the higher-velocity stars are biased towards the right side of the isochrones. This is the locus encompassed by the color and magnitude limits expected for binary stars. In the composite CMD of Group B (right panel) a similar behavior is seen, and stars around the $3.5 \mathrm{Gyr}$ isochrone suggest that higher-velocity stars tend to replace the single-star MS. This loss of single-stars to the field would tend to cause a higher fraction of binaries in such OCRs, in agreement with

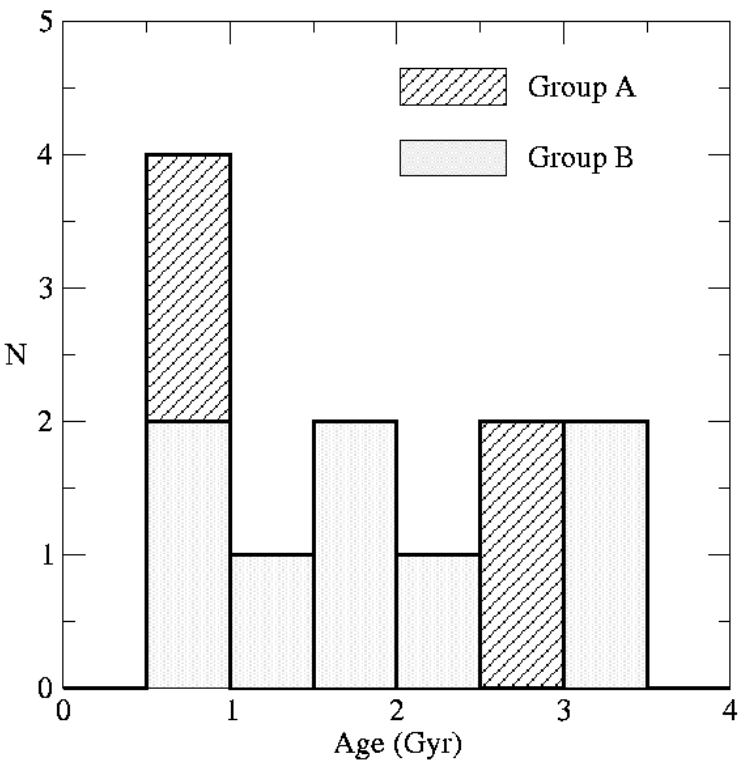

Fig. 14. Age histogram for OCRs and POCRs.

the results of $N$-body simulations (de La Fuente Marcos 1997, 1998).

The composite diagrams of Groups A and B suggest possible evolutionary stages among the studied remnants, where Group B would be more dynamically evolved than Group A, and in general loose objects would be less evolved than compact ones.

\section{Discussion}

The present set of methods for the study of POCRs provided a view of their properties and criteria for their characterization. Of the 18 sample objects, ages, reddening values, and distances could be determined for 16 of them having $I_{\mathrm{f}}>50 \%$ (Table 3). In Table 5 we present a final POCR classification according to different criteria: (i) radial stellar density profile considering Poisson errors; (ii) probability of less than $15 \%$ for the POCR CMD to be representative of the offset field CMD; 

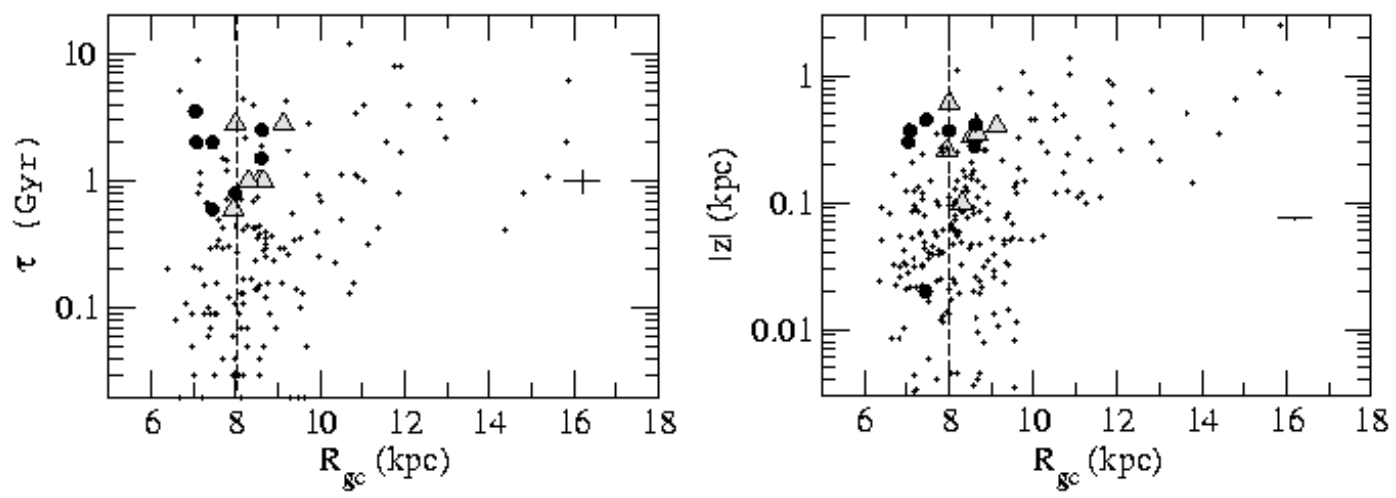

Fig. 15. Age of OCRs and POCRs vs. Galactocentric distance $R_{\mathrm{gc}}$ (left panel), and height from the plane $|z|$ vs. Galactocentric distance $R_{\mathrm{gc}}$ (right panel). The Sun is at $8.0 \mathrm{kpc}$. We distinguish Group A (triangles) and Group B objects (filled circles). Open clusters from Chen et al. (2003) are shown (plus sign).

(iii) homogeneous distribution of stars in the CMD and fitting index $I_{\mathrm{f}} \geq 55 \%$. Columns give the partial classifications in each method, where " $y$ " stands for the attained minimum value, while " $n$ " stands for those not attained. The final results are indicated in the last column, where "OCR" is an object we concluded is an open cluster remnant, FF a field fluctuation, and POCR is maintained as a possible open cluster remnant.

Structural and CMD analyses together are powerful tools for inferring the nature of POCRs. As an example, although NGC 6994 has a reasonable density profile, it has heterogeneous distribution of stars in the CMD (Fig. 6), strongly suggesting a non-physical object.

The statistical method comparing object and field CMDs has provided complementary results. It tests whether an object has a CMD compatible with that of the field. We conclude that $72 \%$ of the studied objects have a probability $p<2 \%$ of being representative of the field (Table 2).

Proper motions are indicators of the internal kinematics of the studied objects. Since we are dealing with intrinsically underpopulated stellar systems, it is important to build composite CMDs and proper-motion diagrams. The OCR composite CMDs show similarities with those of individual open clusters. The OCR composite proper-motion diagrams suggest their classification into two distinct patterns: Group A with one and Group B with two pronounced velocity peaks. The distinction between binary and single stars and their mapping in the CMD suggest evolutionary stages.

Figure 14 shows the age distribution of OCRs and POCRs with $I_{\mathrm{f}}>50 \%$ separated in Groups A and B. The peak of the distribution is in the range $0.5-1.0 \mathrm{Gyr}$, but older objects also exist. Figure 15 shows the objects in Groups A and B in terms of age as a function of Galactocentric distance $R_{\mathrm{gc}}$ (left panel) and height from the plane $|z|$ as a function of $R_{\mathrm{gc}}$ (right panel). For comparison we included 230 open clusters from Chen et al. (2003). The Sun is assumed to be at $8 \mathrm{kpc}$ (Reid 1993). Despite the small sample and the error bars, if we consider that the Group B objects tend to be located inside the Solar circle, this configuration could suggest that tidal effects related to $R_{\mathrm{gc}}$ affect the evolution of remnants (Table 3$)$. Considering a shorter distance $\left(R_{\mathrm{gc}}=\right.$ $7.2 \mathrm{kpc}$ ) of the Sun to the Galactic center (Bica et al. 2006), tidal effects would be stronger.

\section{Concluding remarks}

We studied 18 POCRs by means of 2MASS $J$ and $H$ photometry and UCAC2 proper motions. We analyzed structural properties and distributions of stars in CMDs of POCRs and offset fields using a statistical method. Ages were derived, together with reddening values and distances to the Sun, using the CMDs and Padova isochrones. We defined an index of isochrone fit. All these methods are useful criteria for remnant characterization. The analysis indicates the presence of 13 open cluster remnants in the sample (Table 5).

Open clusters are known to contain a significant fraction of multiple systems as a consequence of their dynamical evolution. The fraction of multiple systems increases and tends to concentrate in the central regions, thus changing the initial spatial distribution of stars (Takahashi \& Portegies Zwart 2000). Numerical simulations show that open cluster remnants are expected to be rich in binary stars. As an example, de La Fuente Marcos (1997) stopped simulations when the cluster population was 10 stars and the number of the remaining binaries was 3 or 4 . Recent studies comparing observational and theoretical results indicate that cluster remnants can form highly hierarchical high-order multiple systems; and considering that the number of such objects can be larger, cluster remnants may be sufficient to account for all observed higher-order multiple systems (Goodwin \& Kroupa 2005).

The present overall results indicate evolutionary differences among sample objects. POCRs show two distinct structures, compact and loose, and we verify a correspondence between the radial stellar density profiles and these structures. Sample objects are basically consistent with OCRs that gradually lose stars as compared to open clusters. The composite proper motion diagrams shows evidence of binary stars, as expected from high binary fractions in dynamically evolved systems. The presence of a sequence of binaries in the composite CMD, replacing the MS of single-stars in Group B, suggests the existence of distinct stages along the dynamical evolution of open cluster remnants. In addition, most of the compact objects are included in Group B and tend to be more internally located in the disc. If two distinct structures might be associated to different evolutionary phases, then transitional objects should be detected. ESO 570 SC12 is visually loose (Fig. 1) but was compact in the radial density profile (Fig. 3). However, a large sample would be necessary to constrain such possible links. For the moment we cannot exclude the possibility that Groups A and B might be descendants of different types of open clusters.

For the first time a study presents a considerable number of POCRs analyzed by means of the same methods for the characterization of their common properties. We verify that is necessary to combine different methods in order to constrain the object 
nature owing to data incompleteness, observational uncertainties, and intrinsically low statistics. The existence of compact and loose POCRs suggests relatively stable remnant structures that dynamically survive beyond open clusters.

We confirm NGC 1252, NGC 1901, Ruprecht 3, and NGC 1663 as open cluster remnants, and NGC 6994 as a field fluctuation, in agreement with previous studies (Pavani et al. 2003; Baume et al. 2003; Carraro 2000; Odenkirchen \& Soubiran 2002). Evidence is found that NGC 6481, NGC 6863, ESO 425 SC6, ESO 425 SC15, ESO 426 SC26, ESO 570 SC12, Ruprecht 31, ESO 383 SC10, and Lyngå 8 are also open cluster remnants, while Waterloo 6 is a field fluctuation. ESO 429 SC2, Object 1, and ESO 132 SC14 are maintained as POCRs for more detailed future studies.

The present work offers observational evidence of the existence in nature of open cluster remnants, as expected from theoretical and numerical studies. As prospective work to answer fundamental questions, we mention (i) would the distinction between compact and loose structures be related to that of the progenitor open cluster and/or the evolutionary processes and (ii), if evolutionary stages in remnants are confirmed, what detailed processes would cause them?

Acknowledgements. We thank Dr. Leandro Kerber for support and advice concerning the development of the statistical method (Sect. 3), and Dr. Charles Bonatto for interesting discussions. This work makes use of data products from the Two Micron All Sky Survey, which is a joint project of the University of Massachusetts and Infrared Processing and Analysis/California Institute of Technology, funded by the National Aeronautics and Space Administration and the National Science Foundation. We also made use of UCAC2 data. We acknowledge support from the Brazilian Institutions CNPq and FAPESP.

\section{References}

Alter, G., Balazs, B., Ruprecht, J., \& Vanysek, J. 1970, Catalogue of Star Cluster and Associations, ed. G. Alter, B. Balazs, \& J. Ruprecht (Budapest: Akademiai Kiado)

Anthony-Twarog, B. J., Twarog, B. A., \& Shodhans, S. 1989, AJ, 98, 1634

Bassino, L. P., Waldhausen, S., \& Martínez, R. 2000, A\&A, 355, 138

Baume, G., Villanova, S., \& Carraro, G. 2003, A\&A, 407, 527

Bergond, G., Leon, S., \& Guilbert, J. 2001, A\&A, 377, 462

Benjamin, R. A., Churchwell, E., Babler, B. L., et al. 2003, PASP, 115, 953

Bica, E., \& Bonatto, C. 2005, A\&A, 431, 973

Bica, E., Santiago, B. X., Dutra, C. M., et al. 2001, A\&A, 366, 827
Bica, E., Bonatto, C., Barbuy, B., \& Ortolani, S. 2006, A\&A, 450, 105 Bonatto, C., Bica, E., \& Pavani, D. B. 2004, A\&A, 427, 485

Bonatto, C., \& Bica, E. 2005, A\&A, 437, 483

Bonatto, C., Bica, E., \& Santos Jr., J. F. C. 2005, A\&A, 433, 917

Carraro, G. 2000, A\&A, 357, 145

Carraro, G. 2002, A\&A, 385, 471

Chen, L., Hou, J. L., \& Wang, J. J. 2003, ApJ, 125, 1397

Carraro, G., Dinescu, D. I., Girard, T. M., \& van Altena, W. F. 2005, A\&A, 433, 143

Epchtein, N., de Batz, B., Capoani, L., et al. 1997, The Messenger, 87, 27

Epchtein, N., Deul, E., Derriere, S., et al. 1999, A\&A, 349, 236

de La Fuente Marcos, R. 1997, A\&A, 322, 764

de La Fuente Marcos, R. 1998, A\&A, 333, L23

Dias, W. S., Moitinho, A., \& Lépine, J. R. D. 2002, A\&A, 389, 871

Dutra, C. M, \& Bica, E. 2000, A\&A, 359, 347

Elson, R., Sigurdsson, S., Davis, M., Hurley, J., \& Gilmore, G. 1998, MNRAS, 300,857

Friel, E. D. 1999, A\&SS, 265, 271

Girardi, L., Bertelli, G., Bressan, A., et al. 2002, A\&A, 391, 195

Hsu, L., Noriega-Crespo, A., \& Friel, E. D. 1996, BASS, 28, 1366

Ivanov, V. D., Borissova, J., Pessev, P., Ivanov, G. R., \& Kurtec, R. 2002, A\&A, 394, L1

Kerber, L. O., Javiel, S. C., \& Santiago, B. X. 2001, A\&A, 365, 424

Kerber, L. O., \& Santiago, B. X. 2005, A\&A, 435, 77

Goodwin, S. P., \& Kroupa, P. 2005, A\&A, 439, 565

King, I. 1966, AJ, 71, 64

Lasker, B. M., Sturch, C. R., Lopez, C., et al. 1988, ApJS, 68, 1

Lyngå, G. 1995, Open Cluster Data, 5th Edition VizieR On-line Data Catalog

Mercer, E. P., Clemens, D. P., Meade, M. R., et al. 2005, ApJ, 635, 560

Mighell, K. J., Rich, R. M., Shara, M., \& Fall, S. M. 1996, AJ, 111, 2314

Odenkirchen, M., \& Soubiran, C. 2002, A\&A, 383, 163

Pavani, D. B., Bica, E., Dutra, C. M., et al. 2001, A\&A, 374, 554

Pavani, D. B., Bica, E., Ahumada, A. V., \& Clariá, J. J. 2003, A\&A, 399, 113

Portegies Zwart, S. F., Makino, J., Mcmillan, S. L. W., \& Hut, P. 2002, ApJ, 565, 265

Reylé, C., \& Robin, A. C. 2001, A\&A, 384, 403

Reid, N. 1993, ARA\&A, 31, 345

Rieke, G. H., \& Lebo, M. J. 1985, ApJ, 228, 618

Sandage, A. 1958, Proceedings of a Conference at Vatican Observatory, ed. D. J. K. O'Connell, 5, 41

Santiago, B. X., Elson, R., \& Gilmore, G. 1996, MNRAS, 281, 1363

Skrutskie, M., Schneider, S., Stiening, R., et al. 1997, The Impact of Large Scale Near-IR Sky Surveys, ed. Garzon et al. (The Netherlands: Kluwer), 210, 187

Takahashi, K., \& Portegies Zwart, S. F. 2000, ApJ, 536, 759

Terlevich, E. 1987, MNRAS, 224, 193

Villanova, S., Carraro, G., de la Fuente Marcos, R., \& Stagni, R. 2004, A\&A, 428, 67

Wielen, R. 1971, A\&A, 13, 309

Zacharias, N., Urban, S. E., Zacharias, M., et al. 2004, AJ, 127, 3043 
D. B. Pavani and E. Bica: Characterization of open cluster remnants, Online Material $p 1$

\section{Online Material}


D. B. Pavani and E. Bica: Characterization of open cluster remnants, Online Material p 2
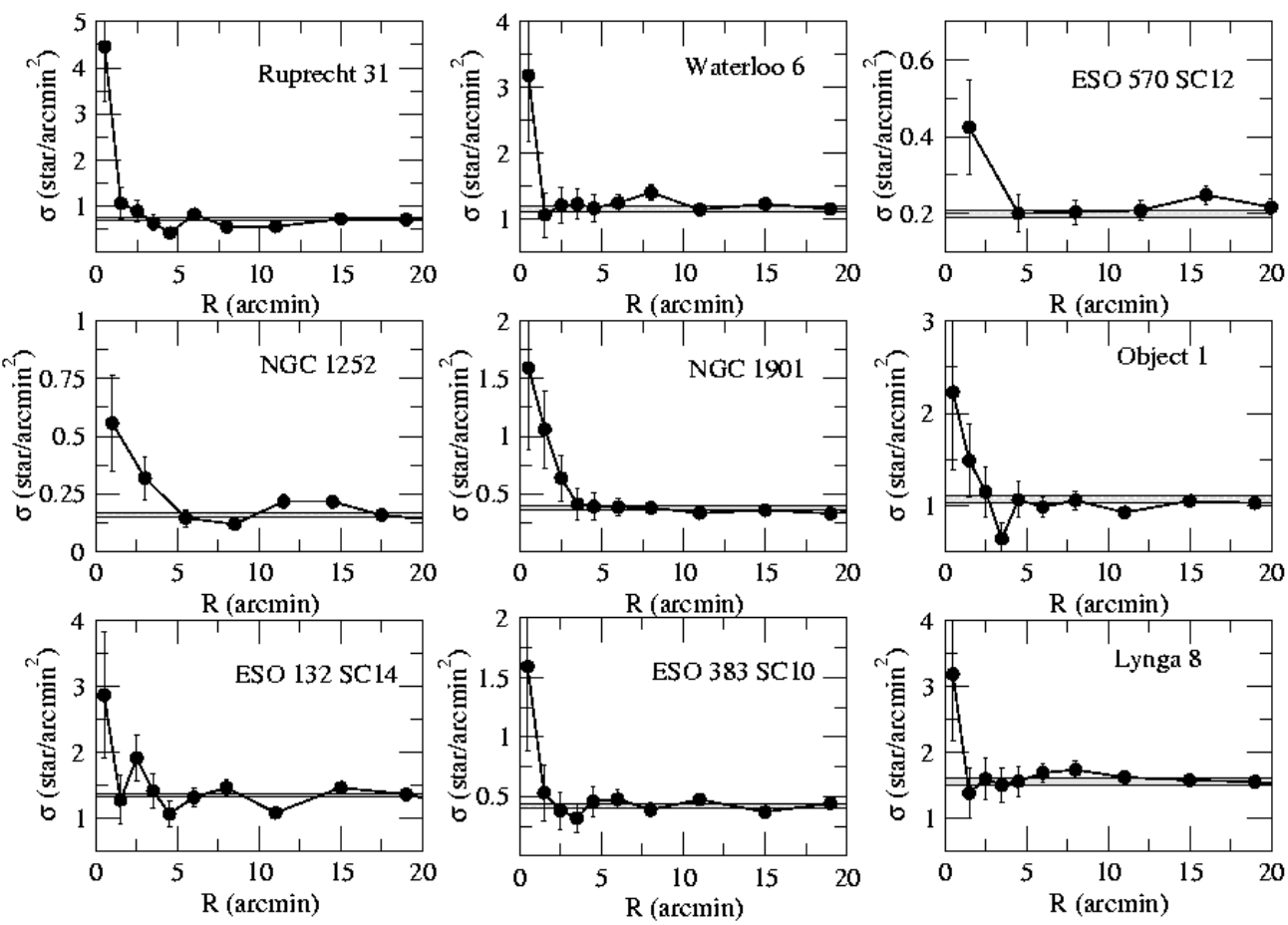

Fig. 3. Same as Fig. 2.

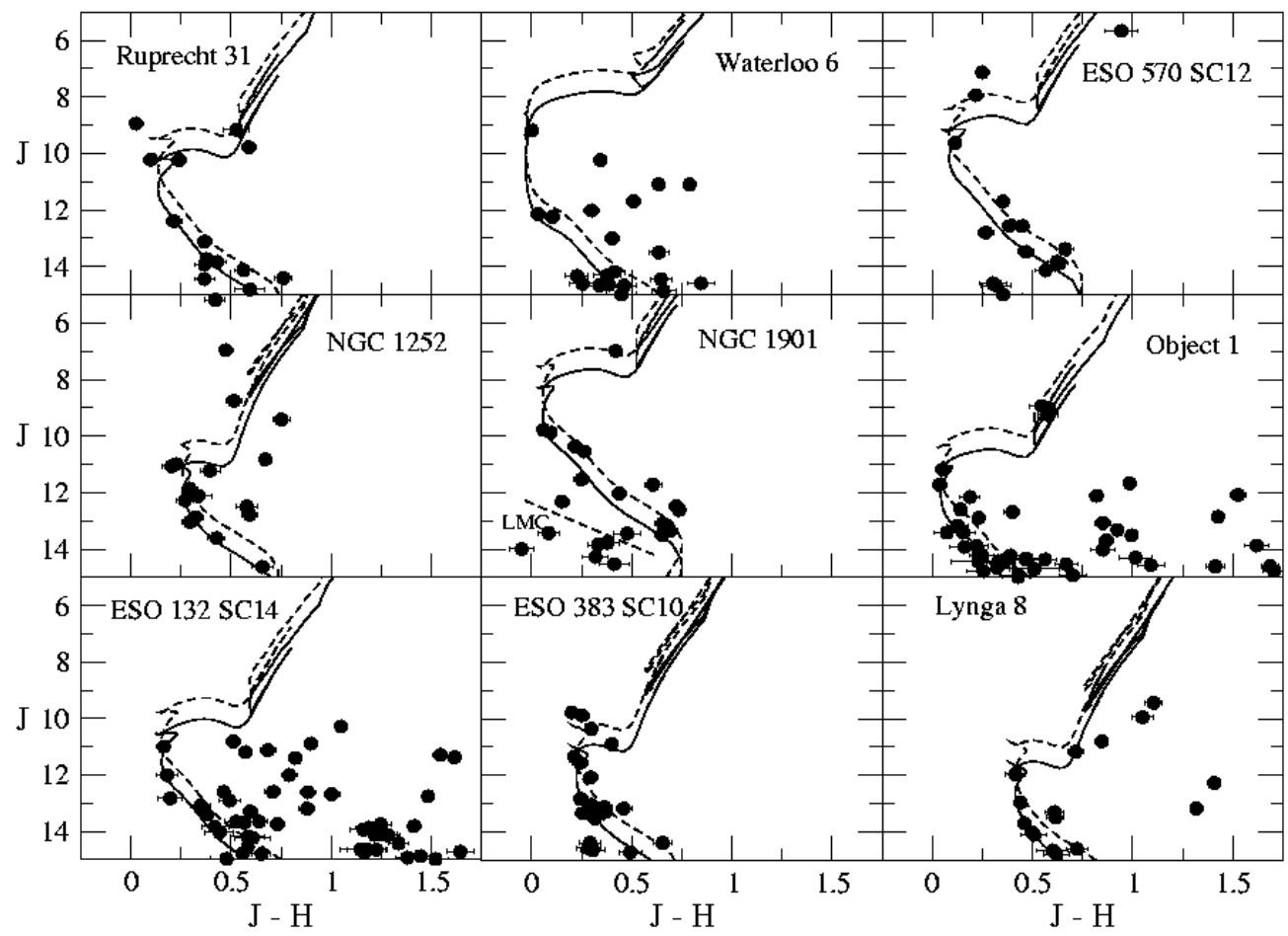

Fig. 7. Same as Fig. 6 for additional POCRs. 
D. B. Pavani and E. Bica: Characterization of open cluster remnants, Online Material p 3

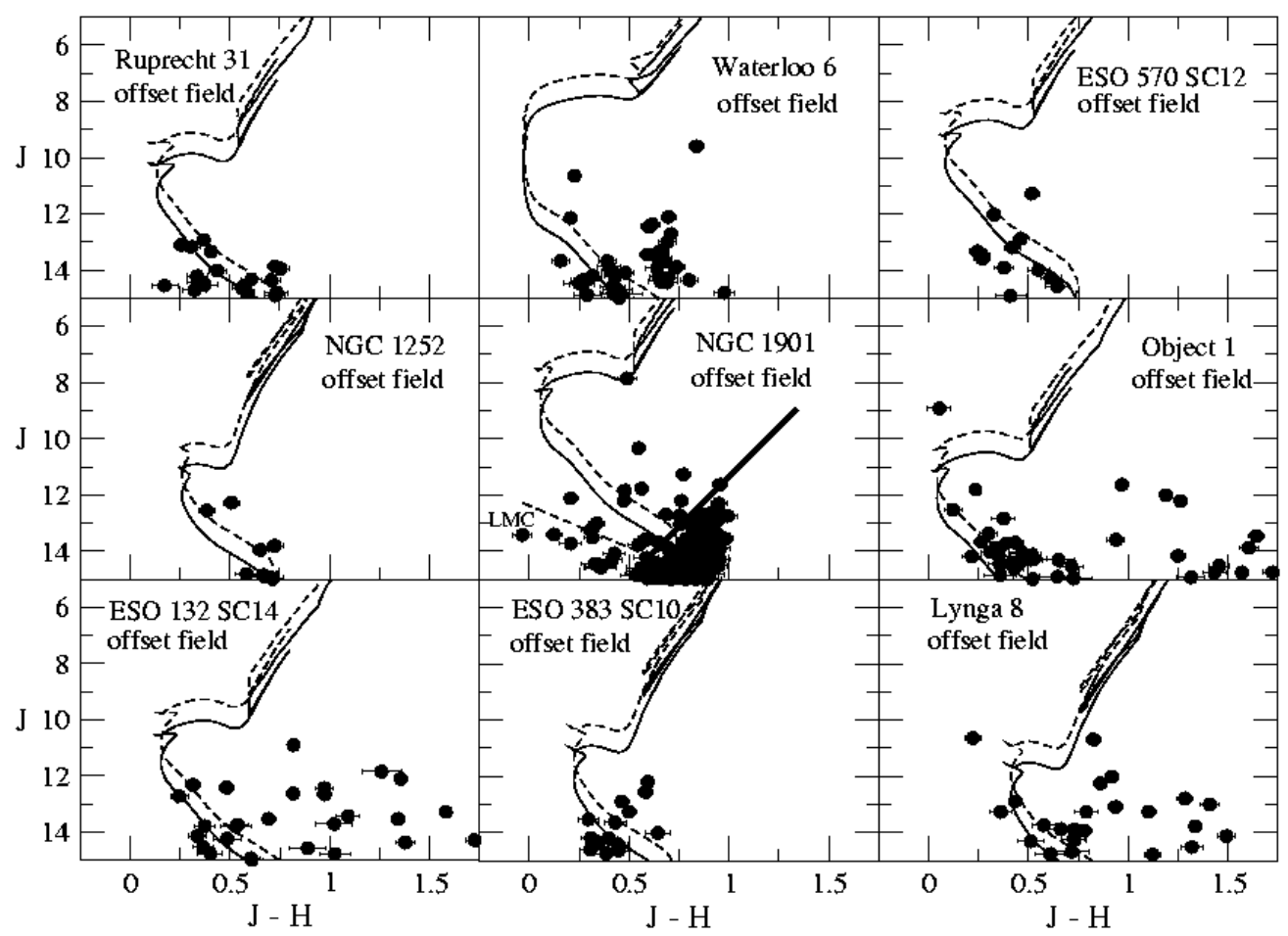

Fig. 9. Same as Fig. 6 for additional offset fields. The thick line in the NGC 1901 CMD indicates contamination of LMC stars eliminated by color filters.

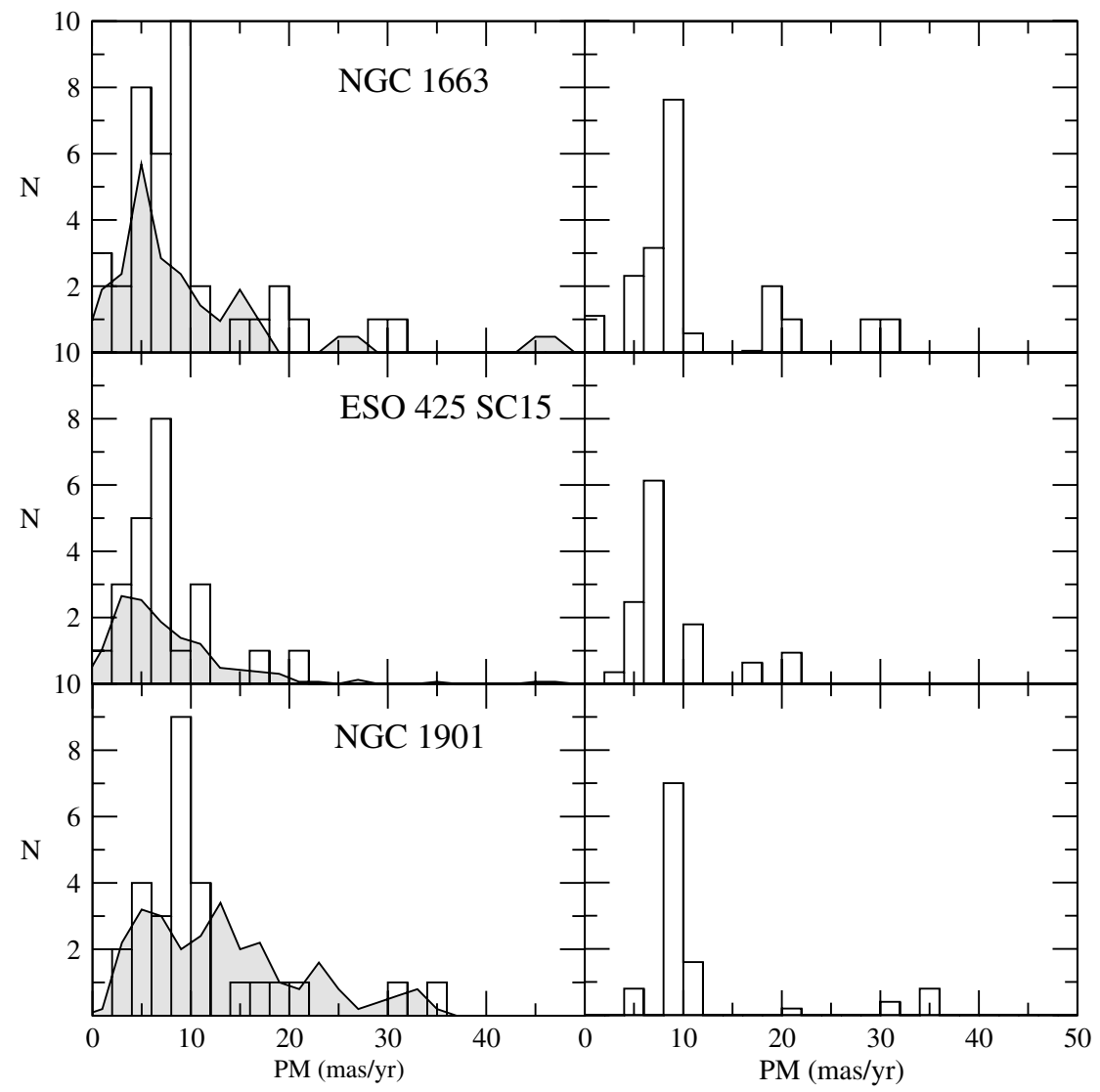

Fig. 10. Left panel: we illustrate the modulus of the proper motion distribution for the object region (histogram) and offset field (shaded area). Right panel: modulus of the proper motion distribution after field subtraction: these POCRs are characterized by a well-defined peak. 